NBER WORKING PAPER SERIES

\title{
EQUITY PREMIA WITH BENCHMARK LEVELS OF CONSUMPTION: CLOSED-FORM RESULTS
}

\author{
Andrew B. Abel \\ Working Paper 12290 \\ http://www.nber.org/papers/w12290 \\ NATIONAL BUREAU OF ECONOMIC RESEARCH \\ 1050 Massachusetts Avenue \\ Cambridge, MA 02138 \\ June 2006
}

The paper was prepared as a chapter in Rajnish Mehra and Edward Prescott (eds.), Handbook of Investments: Equity Risk Premium. It previously circulated under the title "Equity Premia with Benchmark Levels of Consumption, Leverage, Imperfect Correlation of Consumption and Dividends, and Distorted Beliefs: Closed-Form Results.” I thank Martin Lettau, Sydney Ludvigson, Raj Mehra, Jessica Wachter, Amir Yaron, Jianfeng Yu, participants in the Finance Seminar at the Stern School, the European Summer Symposium in Financial Markets, Gerzensee, Switzerland and the Penn Macro Lunch Group for helpful comments. The views expressed herein are those of the author(s) and do not necessarily reflect the views of the National Bureau of Economic Research.

(O2006 by Andrew B. Abel. All rights reserved. Short sections of text, not to exceed two paragraphs, may be quoted without explicit permission provided that full credit, including $\odot$ notice, is given to the source. 
Equity Premia with Benchmark Levels of Consumption: Closed-Form Results

Andrew B. Abel

NBER Working Paper No. 12290

June 2006

JEL No. G12

\begin{abstract}
I calculate exact expressions for risk premia, term premia, and the premium on levered equity in a framework that includes habit formation, keeping/catching up with the Joneses, and possible departures from rational expectations. Closed-form expressions for the first and second moments of returns and for the $\mathrm{R}^{2}$ of a regression of stock returns on the dividend-price ratio are derived under lognormality for the case that includes keeping/catching up with the Joneses. Linear approximations illustrate how these moments of returns are affected by parameter values and illustrate quantitatively how well the model can account for values of the equity premium, the term premium, and the standard deviations of the riskless return and the rate of return on levered equity. For empirically relevant parameter values, the linear approximations yield values of the various moments that are close to those obtained from the exact solutions.
\end{abstract}

Andrew B. Abel

Wharton School

University of Pennsylvania

2315 Steinberg-Dietrich Hall

Philadelphia, PA 19104-6367

and NBER

abel@wharton.upenn.edu 
Mehra and Prescott (1985) showed that over a period of almost a century, the equity premium-the excess of the rate of return on stocks over the rate of return on riskless bills-averaged $6.18 \%$ per year. They then calibrated a general equilibrium asset-pricing model of the sort introduced by Lucas (1978) and showed that such a model, with conventional values of the coefficient of relative risk aversion, cannot come close to accounting for the historically observed equity premium. In the two decades since Mehra and Prescott proclaimed an equity premium puzzle, a large body of research has been devoted to closing the gap between theoretical asset-pricing models and empirically observed asset returns. The seminal study by Mehra and Prescott used a simple general equilibrium model with a representative consumer with constant relative risk aversion transacting in frictionless asset markets to determine the prices of unlevered equity and riskless bills. Subsequent research has extended this simple model to allow for heterogeneous consumers, trading frictions, more general preferences, leverage, a richer time-series of the endowment of consumption, and departures from rational expectations. Since the equity premium puzzle is a quantitative puzzle, many of these studies provide numerical solutions rather closed-form solutions for equilibrium prices and rates of return.

In this chapter, I will provide closed-form solutions for the equilibrium price and rate of return on a canonical asset. The canonical asset is general enough to include riskless bills and risky stocks, and thus allows analysis of the equity premium. I will extend the basic framework used by Mehra and Prescott to allow for preferences that display habit formation and a specific form of externalities. I will also allow for leverage so that I can examine the return on levered equity. Finally, I will allow for departures from rational expectations, though I will set up the framework so that rational expectations is a special-indeed focal-case. Instead of enriching the time-series structure used by Mehra and Prescott, I will simplify it by restricting attention to growth rates of consumption and dividends that are i.i.d. over time, though unlike Mehra and Prescott, I will allow consumption and dividends to differ from each other. As in Mehra and Prescott, I will assume that there is a representative consumer who transacts in frictionless markets.

An advantage of closed-form solutions is that they provide a precise description of how equilibrium rates of return depend on various parameters. Though this description is precise, it is not always transparent. To help understand the effects of various parameters on the equilibrium rates of return, I will provide linear approximations to the closed-form solutions. I will 
show that, for empirically relevant parameter values, these linear approximations tend to be very close to the values obtained by evaluating the exact closed-form expressions.

In this chapter, the major departure from the standard $\operatorname{model}^{1}$ is the specification of preferences to include a benchmark level of consumption that captures habit formation and consumption externalities that are sometimes called "keeping up with the Joneses" or "catching up with the Joneses." I will introduce this more general specification of preferences in Section 1. In Section 2, I introduce the canonical asset and compute its equilibrium price and rate of return. The expression for the rate of return on the canonical asset can be used to determine rates of return on riskless bills, levered equity, and a claim on a single dividend to be received one period in the future. In Section 3, I use these rates of return to define and compute the risk premium, term premium, and equity premium. Beginning in Section 4, I restrict attention to lognormally distributed growth rates. In Section 4, I provide identities that will be helpful in computing expected returns under lognormality. Beginning in Section 5, I generally restrict attention to the case without habit formation, though I allow consumption externalities of the keeping/catching up with the Joneses variety. I compute closed-form expressions for risk, term, and equity premia in Section 5, and in Section 6, I compute linear approximations to these expressions. In Section 7, I compute exact closed-form expressions for the variances of the rates of return, and then I compute linear approximations to these expressions. Although the vector of exogenous growth rates of consumption and dividends is independently and identically distributed over time, the rate of return on stocks is predictable, if preferences display the catching up with the Joneses feature. In fact, the dividend-price ratio can be used to predict the rate of return on stocks, and in Section 8, I compute the $R^{2}$ of a regression of stock returns on the dividend-price ratio. In Section 9, I examine various special cases of the model and focus on the quantitative predictions for the equity premium, the term premium, the standard deviations of the riskless rate and the rate of return on stock, and the $R^{2}$ of the regression of stock returns on the dividend-price ratio. The quantitative implications in Section 9 are based on the approximations of these features of assets, so I show in Section 10 that

\footnotetext{
${ }^{1}$ In this context, the standard model is the Lucas (1978) fruit-tree model, generalized by Mehra and Prescott (1985) to be stationary in growth rates of consumption. The standard model has a representative agent with time-separable utility who can trade assets frictionlessly without restrictions, such as short sale constraints.
} 
the approximations are, in fact, close to the values obtained by evaluating the exact closed-form solutions.

\section{Preferences}

Consider a discrete-time economy with a continuum of identical infinitelylived consumers. Each period the economy receives an endowment of a homogeneous nonstorable good. Because the good is nonstorable, in equilibrium all of it is consumed in the period in which it arrives. The quantity of this good is exogenous and stochastic.

The representative consumer maximizes a utility function that differs in two important ways from the standard time-separable isoelastic utility function commonly used in asset-pricing models. First, the utility flow in each period depends on a benchmark level of consumption that evolves endogenously over time. Second, the representative consumer does not necessarily have rational expectations and thus may not use the objective distributions of random variables when evaluating lifetime utility. For the sake of obtaining closed-form solutions for asset prices, I specify a simple parametric utility function that embodies these two departures from standard preferences. At time $t$, an individual consumer maximizes

$$
U_{t}=E_{t}^{*}\left\{\sum_{j=0}^{\infty}\left(\frac{1}{1+\delta}\right)^{j} \frac{1}{1-\alpha}\left(\frac{c_{t+j}}{\nu_{t+j}^{\gamma}}\right)^{1-\alpha}\right\},
$$

where $c_{t+j}$ is the individual's own consumption in period $t+j, \nu_{t+j}$ is the benchmark level of consumption that I will specify in equation (2), $0 \leq \gamma \leq 1$, the operator $E_{t}^{*}\{\}$ is the conditional expectation based on the consumer's subjective distribution of exogenous stochastic variables as of time $t, \delta \geq 0$ is the rate of time preference, and $\alpha>0$ is a curvature parameter.

Beginning in Section 5, I will confine attention to cases in which the benchmark defined in equation (2) does not depend on a consumer's own past consumption; in these cases, $\alpha$ is the coefficient of relative risk aversion. In the special case in which $\gamma=0$ and in which the subjective distributions equal the true objective distributions, the utility function in equation (1) is the standard time-separable isoelastic utility function with rational expectations.

The parameter $\gamma$ measures the extent to which utility flow in period $t$ depends on $\frac{c_{t}}{\nu_{t}}$, the ratio of the consumer's own consumption to the benchmark 
level of consumption $\nu_{t}$. Specifically, utility flow in period $t$ is an isoelastic function of $\frac{c_{t}}{\nu_{t}^{\gamma}}$, which can be written as an isoelastic function of $c_{t}^{1-\gamma}\left(\frac{c_{t}}{\nu_{t}}\right)^{\gamma}$. Thus, utility flow in period $t$ is an isoelastic function of a weighted geometric average of $c_{t}$, the consumer's own consumption in period $t$, and $\frac{c_{t}}{\nu_{t}}$, the ratio of the consumer's own consumption to the benchmark level of consumption. In the extreme case in which $\gamma=1$, utility flow in period $t$ depends only on the ratio of the consumer's own consumption to the benchmark level of consumption. $^{2,3}$

Suppose that the benchmark level of consumption in period $t$ is

$$
\nu_{t} \equiv c_{t-1}^{D}\left(C_{t}^{\theta_{0}} C_{t-1}^{\theta_{1}} G^{t}\right)^{1-D},
$$

where $c_{t-1}$ is the individual's own consumption in the previous period, $C_{t}$ is the average consumption per person in the current period, $C_{t-1}$ is the average consumption per person in the previous period, $0 \leq D \leq 1, \theta_{0} \geq 0$, $\theta_{1} \geq 0, \theta_{0}+\theta_{1} \leq 1$, and $G \geq 1$. The assumption that $G \geq 1$ allows for the possibility that the benchmark level of consumption can grow exogenously. In equilibrium in this representative consumer economy, the individual's own consumption in period $t, c_{t}$, equals the average consumption per person in period $t, C_{t}$. While an individual consumer treats own consumption, $c_{t}$, as a choice variable, the average consumption per person, $C_{t}$, is exogenous to the individual, and thus is treated as a parameter in the individual's decision problem. If $D=1$, then the benchmark is simply $\nu_{t} \equiv c_{t-1}$,

\footnotetext{
${ }^{2}$ The benchmark, $\nu_{t}$, affects utility flow in period $t$ in the "ratio form" $\frac{c_{t}}{\nu_{t}^{\gamma}}$, rather than in the "difference form" $c_{t}-\nu_{t}^{\gamma}$ as in Constantinides (1990) and Campbell and Cochrane (1999). The ratio form facilitates the finding of closed-form expressions for equilibrium asset prices and returns. As Campbell and Cochane point out, the difference form of the specification allows the coefficient of relative risk aversion to vary over time, whereas the ratio form does not allow this variation. However, the difference form requires that the current level of consumption never falls below the current benchmark level, which requires additional restrictions on the processes generating consumption and the benchmark.

${ }^{3}$ In an economy with production and capital accumulation (Abel (2005)), I rule out the case in which $\gamma=1$. If $\gamma=1$, consumers would be indifferent between a given sequence of consumption and benchmarks $\left\{c_{t}^{*}, \nu_{t}^{*}\right\}_{t=-\infty}^{\infty}$ and, for instance, a sequence $\left\{2 c_{t}^{*}, 2 \nu_{t}^{*}\right\}_{t=-\infty}^{\infty}$ in which all consumptions and benchmarks are twice as high. That is, if $\gamma=1$, consumers are indifferent to the scale of the economy. In a production economy with capital accumulation, the scale of the economy is endogenous, and the indifference to scale would create a problem for a benevolent social planner. However, such a problem does not arise in the endowment economy I study here because the scale of the economy is exogenous.
} 
which is an example of habit formation. In this case, an individual's own consumption in period $t$ directly affects utility flow in period $t$ and also affects the benchmark level of consumption in period $t+1$. (I will use the term "habit formation" to indicate that the benchmark depends on that person's own past consumption. Campbell and Cochrane (1999) have extended this terminology to include cases in which the benchmark in a person's utility function depends on past levels of aggregate consumption per capita, but not directly on past levels of the person's own consumption. They recognize that this terminology is unusual and so refer to such preferences as displaying "external habit formation." I will reserve the term habit formation for cases in which the benchmark depends on the consumer's own past consumption. I will use the term "consumption externalities" to refer to what Campbell and Cochrane call external habit formation. $)^{4}$

The case without habit formation, but with consumption externalities, is represented by $D=0$, so that the benchmark is $\nu_{t} \equiv C_{t}^{\theta_{0}} C_{t-1}^{\theta_{1}} G^{t}$, which is exogenous to an individual consumer. The term $C_{t}^{\theta_{0}} C_{t-1}^{\theta_{1}}$ captures the notion that the benchmark level of consumption depends on the current and/or recent level of consumption per person in the economy. For instance, if $\theta_{0}+\theta_{1}=1$ and $G=1$, the benchmark level of consumption is $\nu_{t}=C_{t}^{\theta_{0}} C_{t-1}^{1-\theta_{0}}$, which is simply a weighted geometric average of current and lagged consumption per person. Within this restricted class of benchmark functions, if $\theta_{0}=1$, so that $\theta_{1}=0$, the benchmark level of consumption is simply $\nu_{t}=C_{t}$ as in Gali (1994). Alternatively, if $\theta_{1}=1$, so that $\theta_{0}=0$, then the benchmark level of consumption is $\nu_{t}=C_{t-1}$, as in the specification of the "catching up with the Joneses" preferences in Abel (1990). ${ }^{5}$

As a step toward calculating the pricing kernel, which is the equilibrium

\footnotetext{
${ }^{4}$ See also the usage in the chapter by George Constantinides in this volume, where he distinguishes between "internal" and "external" habit.

${ }^{5}$ In Abel (1990) I used the term "catching up with the Joneses" to describe the case in which $\nu_{t}=C_{t-1}$, because the benchmark level of consumption depends on lagged rather than current consumption. With this terminology, one might describe the case in which $\nu_{t}=C_{t}$ as a case of "keeping up with the Joneses," because the benchmark level of consumption depends on the contemporaneous consumption of others. Dupor and Liu (2002) refine the terminology for various features of consumption externalities. If utility flow is $u(c, b)$, where $c$ is the individual's own consumption and $b$ is a benchmark that equals the average level of consumption by others, they define jealousy as $\partial u / \partial b<0$ and keeping up with the Joneses as $\partial^{2} u / \partial c \partial b>0$. The utility function in equation (1) displays jealousy. It also displays keeping up with the Joneses for the case in which the curvature parameter $\alpha$, introduced in equation (1), is greater than one.
} 
value of the representative consumer's marginal rate of substitution between current consumption, $c_{t}$, and next period's consumption, $c_{t+1}$, differentiate equation (1) with respect to $c_{t}$ to obtain

$$
\frac{\partial U_{t}}{\partial c_{t}}=\frac{1}{c_{t}} E_{t}^{*}\left\{\left(\frac{c_{t}}{\nu_{t}^{\gamma}}\right)^{1-\alpha}-\gamma D \frac{1}{1+\delta}\left(\frac{c_{t+1}}{\nu_{t+1}^{\gamma}}\right)^{1-\alpha}\right\} .
$$

Because all consumers are identical, $c_{t}=C_{t}$ in equilibrium. Let $x_{t+1} \equiv$ $\frac{C_{t+1}}{C_{t}}=\frac{c_{t+1}}{c_{t}}$ be the (gross) growth rate of consumption from period $t$ to period $t+1$, and use this definition of $x_{t+1}$ and the definition of the benchmark level of consumption in equation (2) to rewrite equation (3) as

$$
\frac{\partial U_{t}}{\partial c_{t}}=c_{t}^{-\alpha} \nu_{t}^{\gamma(\alpha-1)} H_{t}
$$

where

$$
H_{t} \equiv 1-D \gamma \beta E_{t}^{*}\left\{x_{t+1}^{1-\eta}\right\} x_{t}^{v},
$$

and $^{6}$

$$
\begin{aligned}
\beta & \equiv \frac{1}{1+\delta} G^{(1-D) \gamma(\alpha-1)} \\
\eta & \equiv \alpha+\theta_{0}(1-D) \gamma(1-\alpha)>0 \\
v & \equiv \gamma(\alpha-1)\left[D+\theta_{1}(1-D)\right]>-1 .
\end{aligned}
$$

Note that the factor $\beta$ can be larger than one. However, $\beta$ cannot be so large that certain series fail to converge. I will provide a more specific upper bound for $\beta$ in Section 2.1.

The pricing kernel is the intertemporal marginal rate of substitution,

$$
M_{t+1} \equiv \frac{1}{1+\delta} \frac{\partial U_{t+1} / \partial c_{t+1}}{\partial U_{t} / \partial c_{t}} .
$$

\footnotetext{
${ }^{6}$ The inequalities in equations (7) and (8) are derived as follows. Rewrite $\eta$ as $\eta=$ $\left[1-\theta_{0}(1-D) \gamma\right] \alpha+\left[\theta_{0}(1-D) \gamma\right]$. The assumptions that $\theta_{0} \geq 0, \theta_{1} \geq 0$, and $\theta_{0}+\theta_{1} \leq 1$ imply that $0 \leq \theta_{0} \leq 1$ and $0 \leq \theta_{1} \leq 1$. Also, since $0 \leq D \leq 1$ and $0 \leq \gamma \leq 1$, both of the terms in square brackets in the expression for $\eta$ are non-negative, and since the terms in square brackets sum to one, at least one of them must be positive. Since $\alpha>0$, it follows that $\eta>0$.

Rewrite $v$ as $v=\alpha \gamma\left[D+\theta_{1}(1-D)\right]-\gamma\left[D+\theta_{1}(1-D)\right]$, so that $v+1=$ $\left\{\alpha \gamma\left[D+\theta_{1}(1-D)\right]\right\}+\left\{1-\gamma\left[D+\theta_{1}(1-D)\right].\right\} \quad$ Since $0 \leq D \leq 1,0 \leq \gamma \leq 1$, and $0 \leq \theta_{1} \leq 1$, it follows that $0 \leq \gamma\left[D+\theta_{1}(1-D)\right] \leq 1$. Therefore, since $\alpha>0$, both of the terms in curly brackets in the expression for $v+1$ are non-negative and at least one of them is positive. Therefore, $v+1>0$, so $v>-1$.
} 
Use equation (4) and the definition of the benchmark in equation (2) to rewrite equation (9) as

$$
M_{t+1}=\beta x_{t}^{v} x_{t+1}^{-\eta} \frac{H_{t+1}}{H_{t}} .
$$

In the standard case of time-separable isoelastic utility, which is represented by $\gamma=0$, we have $\beta=\frac{1}{1+\delta}, \eta=\alpha, v=0$, and $H_{t} \equiv 1$. Therefore, the marginal rate of substitution in equation (10) is simply

$$
M_{t+1}=\beta x_{t+1}^{-\alpha}, \text { if } \gamma=0 .
$$

If we introduce a benchmark level of consumption into the utility function $(\gamma>0)$, but confine attention to the case with $D=0$, so that there may be consumption externalities but no habit formation, the marginal rate of substitution in equation (10) is

$$
M_{t+1}=\beta x_{t}^{v} x_{t+1}^{-\eta}, \text { if } D=0
$$

since $H_{t} \equiv 1$ when $D=0$. If the growth rate of consumption, $x_{t}$, is independently distributed over time, then in the standard isoelastic case, $M_{t+1}=\beta x_{t+1}^{-\alpha}$ is also independently distributed over time. However, with a benchmark level of consumption, the pricing kernel $M_{t}$ in equation (12) is, in general, not independently distributed over time. In fact, since $\eta>0$, the serial correlation of $M_{t}$ has the opposite sign of $v$, which can be positive, negative or zero. Under rational expectations, Backus, Gregory, and Zin (1989) have shown that the term premium has the opposite sign of the serial correlation of the pricing kernel. Therefore, the term premium has the same sign as $v$, as I will show under lognormality of $x_{t}$ (and without habit formation) in Section 5. In the standard case of time-separable utility, $v=0$ so the term premium is zero if the growth rate of consumption, $x_{t}$, is independently distributed over time.

In the case considered by Gali (1994) $\left(\theta_{1}=0=D\right.$, so that $v=0$; and $G=1$ ), the marginal rate of substitution in equation (12) becomes $M_{t+1}=$ $\beta x_{t+1}^{-\eta}$, which is the pricing kernel for standard time-separable isoelastic utility in which the coefficient of relative risk aversion is constant and equal to $\eta$. Gali points out an interesting special case in which $\gamma=1=\theta_{0}$, so that the benchmark is simply $\nu_{t}=C_{t}$ and, as in Abel (1990), consumers care only about the ratio of their own consumption to the benchmark. In this special case, $\eta=1$ so that regardless of the value of the curvature 
parameter $\alpha$, the pricing kernel is $M_{t+1}=\beta x_{t+1}^{-1}$, which is the marginal rate of substitution for a standard logarithmic utility function without benchmark levels of consumption $(\alpha=1$ and $\gamma=0)$. Thus, asset prices in this special case of Gali's specificaiton would be identical to asset prices that would arise in the simple case of logarithmic utility without benchmark levels of consumption.

The specification of the utility function has seven fundamental parameters: the curvature paramater $\alpha$, the rate of time preference $\delta$, the importance of the consumption-benchmark ratio as measured by $\gamma$, the relative weight $D$ of the consumer's own past consumption in the benchmark, the weights $\theta_{0}$ and $\theta_{1}$ on contemporaneous and lagged aggregate consumption in the benchmark, and the exogenous growth rate $G$ in the benchmark. Beginning in Section 5, I generally restrict attention to the case without habit formation, though I allow consumption externalities of the keeping/catching up with the Joneses variety. In this case, $D=0$, which implies $H_{t} \equiv 1$. With $H_{t} \equiv 1$, equation (10) shows that the pricing kernel depends on only the three "derived" preference parameters $\beta, \eta$, and $v$ in equations (6), (7), and (8), respectively. Thus, one could not recover the seven fundamental parameters by observing data on asset returns. The reason for starting with seven fundamental parameters is to illustrate that many different representations of preferences can be captured by the three derived parameters $\beta, \eta$, and $v$. These parameters are defined precisely in equations $(6),(7)$, and (8), respectively. Loosely speaking, $\beta$ is an adjusted time-preference discount factor, $\eta$ is a curvature parameter related to risk aversion, and $v$ is a parameter reflecting the role of past (aggregate or individual) consumption. Table 1 summarizes the relationships among the fundamental preference parameters and the derived preference parameters in interesting special cases.

To see how the marginal rate of substitution determines asset prices and rates of return, let $R_{t+1}$ be the one-period gross rate of return on an asset from period $t$ to period $t+1$. Consider a consumer who reduces consumption in period $t$ by one unit and purchases one unit of the asset in period $t$. In period $t+1$, the consumer collects any payoff (for instance, dividends or coupons) and then sells the asset and uses the proceeds to increase consumption in period $t+1$. Since the payoff plus the proceeds from the sale of the asset equal $R_{t+1}$ units of consumption, the consumer can increase $c_{t+1}$ by $R_{t+1}$. Optimality from the consumer's point of view in period $t$ requires that the subjective expected reduction in utility from reducing $c_{t}$ by one unit equals the subjective expected increase in utility from increasing $c_{t+1}$ by $R_{t+1}$ units, 


\begin{tabular}{|c|c|c|c|c|}
\hline & $\begin{array}{c}\text { Time } \\
\text { Separable }\end{array}$ & $\begin{array}{c}\text { Habit } \\
\text { Formation }\end{array}$ & $\begin{array}{c}\text { Keeping Up } \\
\text { with the Joneses }\end{array}$ & $\begin{array}{c}\text { Catching Up } \\
\text { with the Joneses }\end{array}$ \\
\hline \multicolumn{5}{|c|}{ Fundamental Preference Parameters } \\
\hline$\gamma$ & 0 & $0<\gamma \leq 1$ & $0<\gamma \leq 1$ & $0<\gamma \leq 1$ \\
\hline$\delta$ & $\geq 0$ & $\geq 0$ & $\geq 0$ & $\geq 0$ \\
\hline$\alpha$ & $>0$ & $>0$ & $>0$ & $>0$ \\
\hline$G$ & - & - & $\geq 1$ & $\geq 1$ \\
\hline$D$ & - & 1 & 0 & 0 \\
\hline$\theta_{0}$ & - & - & 1 & 0 \\
\hline$\theta_{1}$ & - & - & 0 & 1 \\
\hline \multicolumn{5}{|c|}{ Derived Preference Parameters and Pricing Kernel } \\
\hline$\beta$ & $\frac{1}{1+\delta}$ & $\frac{1}{1+\delta}$ & $\frac{1}{1+\delta} G^{\gamma(\alpha-1)}$ & $\frac{1}{1+\delta} G^{\gamma(\alpha-1)}$ \\
\hline$\eta$ & $\alpha$ & $\alpha$ & $\alpha+\gamma(1-\alpha)$ & $\alpha$ \\
\hline$v$ & 0 & $\gamma(\alpha-1)$ & 0 & $\gamma(\alpha-1)$ \\
\hline$H_{t}$ & 1 & $1-\beta \gamma E_{t}^{*}\left\{x_{t+1}^{1-\alpha}\right\} x_{t}^{\gamma(\alpha-1)}$ & 1 & 1 \\
\hline$M_{t+1}$ & $\beta x_{t+1}^{-\alpha}$ & $\beta x_{t}^{\gamma(\alpha-1)} x_{t+1}^{-\alpha} \frac{H_{t+1}}{H_{t}}$ & $\beta x_{t+1}^{-\alpha-\gamma(1-\alpha)}$ & $\beta x_{t}^{\gamma(\alpha-1)} x_{t+1}^{-\alpha}$ \\
\hline \multicolumn{5}{|c|}{ Special Case in which $\gamma=1$} \\
\hline$H_{t}$ & - & $1-\beta E_{t}^{*}\left\{x_{t+1}^{1-\alpha}\right\} x_{t}^{\alpha-1}$ & 1 & 1 \\
\hline$M_{t+1}$ & - & $\beta x_{t}^{\alpha-1} x_{t+1}^{-\alpha} \frac{H_{t+1}}{H_{t}}$ & $\beta x_{t+1}^{-1}$ & $\beta x_{t}^{\alpha-1} x_{t+1}^{-\alpha}$ \\
\hline
\end{tabular}

Table 1: Preference Parameters and Pricing Kernel in Various Cases 
so that

$$
\frac{\partial U_{t}}{\partial c_{t}}=E_{t}^{*}\left\{R_{t+1} \frac{1}{1+\delta} \frac{\partial U_{t+1}}{\partial c_{t+1}}\right\} .
$$

The first-order condition in equation (13) can be rewritten using the definition of the marginal rate of substitution in equation (9) as

$$
E_{t}^{*}\left\{M_{t+1} R_{t+1}\right\}=1
$$

Equation (14) is the familiar fundamental condition of asset pricing: the product of the marginal rate of subsitution and the gross rate of return on any frictionlessly traded asset has a conditional expectation equal to one. The only unfamiliar aspect of equation (14) is that the conditional expectation is based on the consumer's subjective distribution, which may differ from the objective distribution. Using the marginal rate of substitution in equation (10), the fundamental condition of asset pricing can be written as

$$
\frac{\beta x_{t}^{v}}{H_{t}} E_{t}^{*}\left\{x_{t+1}^{-\eta} H_{t+1} R_{t+1}\right\}=1 .
$$

In the next section, I will define a canonical asset and will use equation (15) to determine the equilibrium price of this asset.

\section{The Canonical Asset}

The fundamental condition for asset pricing in equation (15) can be used to determine the price of any frictionlessly traded asset in the absence of borrowing constraints and other restrictions such as short sale constraints. To avoid the need for separate derivations for the equilibrium prices of, for instance, riskless bills and stocks, I will calculate the equilibrium price of a canonical asset, introduced in Abel (1999), that is general enough to include these assets as special cases.

The canonical asset is an $n$-period asset, where $n$ may be infinite. In period $t$, the canonical asset is a claim to a stream of $n$ payoffs $a_{n-1} y_{t+1}^{\lambda}$, $a_{n-2} y_{t+2}^{\lambda}, \ldots, a_{0} y_{t+n}^{\lambda}$, where $a_{0}>0, a_{j} \geq 0, j=1, \ldots, n-1$ are constants, $y_{t+j}>$ 0 is a random variable, and $\lambda \geq 1$ is a constant. The (gross) growth rate of the random variable $y_{t}$ is $z_{t+1} \equiv \frac{y_{t+1}}{y_{t}}$. Recall that $x_{t+1}$ is the (gross) growth rate of consumption per capita. I will assume that the vector $\left(x_{t+1}, z_{t+1}\right)$ is i.i.d. over time and that even when the subjective distributions differ 
from the corresponding objective distributions, the subjective distributions incorporate the fact that $\left(x_{t+1}, z_{t+1}\right)$ is i.i.d. over time. The definition of $H_{t}$ in equation (5) and the assumption that $\left(x_{t+1}, z_{t+1}\right)$ is i.i.d. over time imply that $\left(x_{t+1}, z_{t+1}, H_{t+1}\right)$ is i.i.d. over time.

When $\lambda=0$, the canonical asset is a fixed-income security, with a deterministic stream of payoffs $a_{n-j}$ in period $t+j$, for $j=1, \ldots, n$. For instance, a coupon bond with face value $F$, maturity $n$, and coupon $d$ is represented by $a_{0}=d+F$ and $a_{j}=d$, for $j=1, \ldots, n-1$. A pure discount bond is represented by $d=0$. A consol that pays a constant coupon $d$ forever is represented by $a_{j}=d$, for $j=1,2,3, \ldots$.

Securities with risky payoffs are represented by $\lambda \neq 0$. For instance, in Lucas (1978), unlevered equity pays a dividend per capita in each period that equals consumption per capita. Thus the payoff in period $t+j$ is $C_{t+j}$, so unlevered equity can be represented by the canonical asset with $\lambda=1, n=\infty, a_{j}=1$, and $y_{t+j} \equiv C_{t+j}$, for $j=1,2,3, \ldots$ As explained in Abel (1999), levered equity is represented by $\lambda>1 .^{7}$ This formulation of levered equity has been adopted by, for example, Bansal and Yaron (2003) and Lettau, Ludvigson, and Wachter (2004).

\footnotetext{
${ }^{7}$ To illustrate levered equity most simply, consider a heuristic example with one-period levered equity. Define $q \equiv y-B$, where $y$ is the payoff to owners of equity before making any payment to bondholders, and $0 \leq B<E\{y\}$ is the payment promised to bondholders. The payoff to one-period levered equity is $\max [q, 0]$. If the probability of default is small enough to allow us to ignore default, the payoff to levered equity is simply $q$, with mean $E\{q\}=E\{y\}-B$, standard deviation $s d\{q\}=s d\{y\}$, and coefficient of variation equal

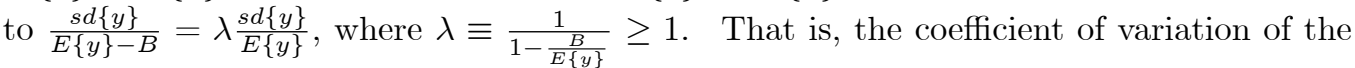
levered payoff (ignoring bankruptcy) is $\lambda$ times as large as the coefficient of variation of the unlevered payoff $y$. If $y$ is lognormally distributed, then the coefficient of variation of $y^{\lambda}$ is approximately $\lambda$ times as large as the coefficient of variation of $y$, so $y^{\lambda}$ with $\lambda>1$ reflects the fact that leverage increases the coefficient of variation of the payoff to equity. If we interpret $\frac{B}{E\{y\}}$ as the leverage ratio, then $\lambda$ is an increasing function of the leverage ratio. Note that without leverage, i.e., with $B=0, \lambda=1$. This description is heuristic and, importantly, has ignored the role of default. In Abel (1999), I take explicit account of bankruptcy, and show that for a broad range of values of the leverage ratio $\frac{B}{E\{y\}}$, the rate of return on a one-period asset paying $y^{\lambda}$ is very close to the rate of return on a one-period asset paying $\max [y-B, 0]$.
} 


\subsection{The Price of the Canonical Asset}

Let $p_{t}(n, \lambda)$ be the ex-payment (e.g., ex-dividend for equity, ex-coupon for bonds) price of the canonical asset in period $t$. The price also depends on the sequence of the constants $a_{j}, j=0, \ldots, n-1$, and on the properties of the stochastic process for $y_{t}$, but, to reduce clutter, I have not included this dependence in the notation. A consumer can buy a unit of the canonical asset at price $p_{t}(n, \lambda)$ in period $t$ and then in period $t+1$ can collect the payoff $a_{n-1} y_{t+1}^{\lambda}$ and sell the asset at price $p_{t+1}(n-1, \lambda)$ when the asset becomes an $(n-1)$-period asset. Therefore, the gross rate of return on the asset from period $t$ to period $t+1$ is

$$
R_{t+1}(n, \lambda)=\frac{a_{n-1} y_{t+1}^{\lambda}+p_{t+1}(n-1, \lambda)}{p_{t}(n, \lambda)} .
$$

Substituting the rate of return on the canonical asset from equation (16) into the fundamental condition of asset pricing in equation (15), recognizing that $p_{t}(n, \lambda)$ is known at time $t$, and multiplying both sides by $p_{t}(n, \lambda)$ yields a recursive expression for the price of the canonical asset

$$
p_{t}(n, \lambda)=\frac{\beta x_{t}^{v}}{H_{t}} E_{t}^{*}\left\{x_{t+1}^{-\eta} H_{t+1}\left[a_{n-1} y_{t+1}^{\lambda}+p_{t+1}(n-1, \lambda)\right]\right\} .
$$

I will hypothesize that the price of the canonical asset, which must satisfy equation (17), is

$$
p_{t}(n, \lambda)=\omega(n, \lambda) \frac{x_{t}^{v} y_{t}^{\lambda}}{H_{t}}
$$

where $\omega(n, \lambda)$ is a function to be determined. As a step toward determining the function $\omega(n, \lambda)$, substitute equation (18) into equation (17) and use the facts that $a_{n-1}$ and $\omega(n-1, \lambda)$ are nonstochastic and that $\left(x_{t}, z_{t}\right)$ is i.i.d. (so that the unconditional means of $x_{t+1}^{v-\eta} z_{t+1}^{\lambda}$ and $x_{t+1}^{-\eta} H_{t+1} z_{t+1}^{\lambda}$ equal the means of the corresponding conditional distributions at time $t$ ) to simplify and obtain

$$
\omega(n, \lambda)=A(\lambda) \omega(n-1, \lambda)+B(\lambda) a_{n-1}, \text { for } n \geq 1,
$$

where

$$
A(\lambda) \equiv \beta E^{*}\left\{x_{t+1}^{v-\eta} z_{t+1}^{\lambda}\right\}>0
$$

and

$$
B(\lambda) \equiv \beta E^{*}\left\{x_{t+1}^{-\eta} H_{t+1} z_{t+1}^{\lambda}\right\}>0
$$


Equation (19) is a first-order linear difference equation. In order to guarantee that this difference equation converges as $n$ grows without bound, I will assume that $\beta$ and the subjective distribution of $\left(x_{t+1}, z_{t+1}\right)$ are such that $A(\lambda)<1$. This assumption is equivalent to $\beta<\left[E^{*}\left\{x_{t+1}^{v-\eta} z_{t+1}^{\lambda}\right\}\right]^{-1}$, which allows $\beta$ to exceed one to the extent to the $E^{*}\left\{x_{t+1}^{v-\eta} z_{t+1}^{\lambda}\right\}$ is less than one.

Equation (19) has a simple boundary condition associated with it. After an asset has paid its last payment, it is formally a zero-period asset, and its price is zero. That is, $p_{t}(0, \lambda)=0$ so $\omega(0, \lambda)=0$. Using this boundary condition, evaluate the difference equation in equation (19) for $n=1$ to obtain

$$
\omega(1, \lambda)=B(\lambda) a_{0}
$$

For arbitrary $n$, the solution to the difference equation in equation (19) that satisfies the boundary condition in equation (22) is

$$
\omega(n, \lambda)=B(\lambda) \sum_{i=1}^{n} a_{i-1}[A(\lambda)]^{n-i} .
$$

The function $\omega(n, \lambda)$ can be increasing or decreasing in the maturity $n$. For a pure discount bond with face value $F, a_{0}=F$ and $a_{j}=0$ for $j=1, \ldots, n-1$. In this case, $\omega(n, \lambda)=B(\lambda)[A(\lambda)]^{n-1} F$, which is strictly decreasing in $n$ because $A(\lambda)<1$. For a security with a level stream of payoff coefficients $a_{j}=d$ for $j=0, \ldots, n-1$, we have $\omega(n, \lambda)=B(\lambda) \frac{1-[A(\lambda)]^{n}}{1-A(\lambda)} d$, which is increasing in the maturity $n$. A coupon bond is a composite of a discount bond with a face value of $F$ and a security that promises a level stream of payments $d$, so $a_{0}=d+F$ and $a_{j}=d$, for $j=1, \ldots, n-1$. Therefore, for an $n$-period coupon bond, $\omega(n, \lambda)=B(\lambda)\left\{[A(\lambda)]^{n-1} F+\frac{1-[A(\lambda)]^{n}}{1-A(\lambda)} d\right\}$, which is increasing in $n$ if $d / F>\frac{1-A(\lambda)}{A(\lambda)}$, decreasing in $n$ if $d / F<\frac{1-A(\lambda)}{A(\lambda)}$, and invariant to $n$ if $d / F=\frac{1-A(\lambda)}{A(\lambda)}$. That is, if the coupon is small relative to the face value, the coupon bond is more like a discount bond in which $\omega(n, \lambda)$ is decreasing in $n$. If the coupon is large relative to the face value, the coupon bond is more like a security with a flat stream of payoffs, and $\omega(n, \lambda)$ is increasing in maturity $n$.

The expression for $\omega(n, \lambda)$ in equation (23) completes the solution of the equilibrium price of the canonical asset for arbitrary values of $n$ and $\lambda$. The functions $A(\lambda)$ and $B(\lambda)$ are unconditional moments of the subjective distributions of exogenous random variables. Given the subjective distributions 
of the exogenous random variables, equations (20) and (21) give the values of $A(\lambda)$ and $B(\lambda)$, which can be used along with the sequence of payoff coefficients $a_{j}, j=0, \ldots, n-1$ in equation (23) to obtain the value of $\omega(n, \lambda)$. Substituting this value of $\omega(n, \lambda)$ into equation (18), and using the definition of $H_{t}$ in equation (5) gives the price of the canonical asset for any $n$ and $\lambda$.

\subsection{The Rate of Return on the Canonical Asset}

To calculate the rate of return on the canonical asset, substitute the expression for the price of the canonical asset from equation (18) into the expression for the rate of return in equation (16) to obtain

$$
R_{t+1}(n, \lambda)=\frac{a_{n-1}+\omega(n-1, \lambda) \frac{x_{t+1}^{v}}{H_{t+1}}}{\omega(n, \lambda)} H_{t} x_{t}^{-v} z_{t+1}^{\lambda} .
$$

It will be convenient to rewrite the rate of return on the canonical asset in equation (24) as

$$
R_{t+1}(n, \lambda)=J\left(x_{t+1}, z_{t+1}, H_{t+1} ; n, \lambda\right) \times K\left(x_{t}, H_{t}\right),
$$

where

$$
J\left(x_{t+1}, z_{t+1}, H_{t+1} ; n, \lambda\right) \equiv \frac{a_{n-1}}{\omega(n, \lambda)} z_{t+1}^{\lambda}+\frac{\omega(n-1, \lambda)}{\omega(n, \lambda)} \frac{x_{t+1}^{v} z_{t+1}^{\lambda}}{H_{t+1}}
$$

and

$$
K\left(x_{t}, H_{t}\right) \equiv x_{t}^{-v} H_{t}
$$

Equation (25) expresses the rate of return on the canonical asset as the product of $J\left(x_{t+1}, z_{t+1}, H_{t+1} ; n, \lambda\right)$ and $K\left(x_{t}, H_{t}\right)$, which are independent of each other because the vector of exogenous random variables $\left(x_{t}, z_{t}, H_{t}\right)$ is independently distributed over time.

I will confine attention to assets for which $a_{j}=1$, for $j=0,1,2, \ldots, n-1$. In this case, equation (23) implies that

$$
\frac{1}{\omega(n, \lambda)}=\frac{1}{B(\lambda)} \frac{1-A(\lambda)}{1-[A(\lambda)]^{n}}
$$

which implies that

$$
\frac{\omega(n-1, \lambda)}{\omega(n, \lambda)}=\frac{1-[A(\lambda)]^{n-1}}{1-[A(\lambda)]^{n}}
$$


Substitute equations (28) and (29) into the definition of $J\left(x_{t+1}, z_{t+1}, H_{t+1} ; n, \lambda\right)$ in equation (26), set $a_{n-1}=1$, and rearrange to obtain

$$
\begin{aligned}
& J\left(x_{t+1}, z_{t+1}, H_{t+1} ; n, \lambda\right) \\
= & \frac{1}{1-[A(\lambda)]^{n}}\left[\frac{1-A(\lambda)}{B(\lambda)} z_{t+1}^{\lambda}+\left(1-[A(\lambda)]^{n-1}\right) x_{t+1}^{v} z_{t+1}^{\lambda} H_{t+1}^{-1}\right] .
\end{aligned}
$$

Because $\left(x_{t}, z_{t}, H_{t}\right)$ is i.i.d. over time, $J\left(x_{t+1}, z_{t+1}, H_{t+1} ; n, \lambda\right)$ is i.i.d. over time. Therefore, the objective conditional expectation of $J\left(x_{t+1}, z_{t+1}, H_{t+1} ; n, \lambda\right)$ equals the objective unconditional expectation of $J\left(x_{t+1}, z_{t+1}, H_{t+1} ; n, \lambda\right)$.

Now define

$$
\Psi(\lambda) \equiv \frac{E\left\{H^{-1} x^{v} z^{\lambda}\right\}}{E\left\{z^{\lambda}\right\}} \frac{B(\lambda)}{A(\lambda)},
$$

where the operator $E\{\}$ (without an asterisk) denotes the expectation using the objective distribution of the relevant random variables. I have suppressed the time subscripts from the variables inside the expectation operator, with the understanding that any variables inside the operator (such as $H$ and $x$ ) are contemporaneous with each other (i.e, $H_{t}$ and $x_{t}$ ). I will show later that $\Psi(\lambda)$ helps determine the term premium.

Use equation (31) to rewrite equation (30) as

$$
\begin{aligned}
& J\left(x_{t+1}, z_{t+1}, H_{t+1} ; n, \lambda\right)=\frac{1}{1-[A(\lambda)]^{n}} \frac{E\left\{z^{\lambda}\right\}}{B(\lambda)} \\
& \times\left[[1-A(\lambda)] \frac{z_{t+1}^{\lambda}}{E\left\{z^{\lambda}\right\}}+\left(1-[A(\lambda)]^{n-1}\right) \Psi(\lambda) A(\lambda) \frac{x_{t+1}^{v} z_{t+1}^{\lambda} H_{t+1}^{-1}}{E\left\{x^{v} z^{\lambda} H^{-1}\right\}}\right] .
\end{aligned}
$$

Now compute the objective unconditional expectations of both sides of equation (32) to obtain

$E\left\{J\left(x_{t+1}, z_{t+1}, H_{t+1} ; n, \lambda\right)\right\}=\left[1+A(\lambda)[\Psi(\lambda)-1] \frac{1-[A(\lambda)]^{n-1}}{1-[A(\lambda)]^{n}}\right] \frac{E\left\{z^{\lambda}\right\}}{B(\lambda)}$.

The expectation of $K\left(x_{t}, H_{t}\right)$ is calculated directly from the definition in equation (27) to obtain

$$
E\left\{K\left(x_{t}, H_{t}\right)\right\} \equiv E\left\{x^{-v} H\right\} .
$$

Finally, calculate the expected value of $R_{t+1}(n, \lambda)$ in equation (25) using the independence of $J\left(x_{t+1}, z_{t+1}, H_{t+1} ; n, \lambda\right)$ and $K\left(x_{t}, H_{t}\right)$ and equations 
(33) and (34) to obtain

$E\left\{R_{t+1}(n, \lambda)\right\}=\left[1+A(\lambda)[\Psi(\lambda)-1] \frac{1-[A(\lambda)]^{n-1}}{1-[A(\lambda)]^{n}}\right] \frac{E\left\{z^{\lambda}\right\}}{B(\lambda)} E\left\{x^{-v} H\right\}$.

Equation (35) gives the unconditional expected rate of return on a canonical asset with arbitary $\lambda$ and arbitrary $n$, provided that $a_{0}=\ldots=a_{n-1}=1$. This expression simplifies considerably for one-period canonical assets. With $n=1$,

$$
E\left\{R_{t+1}(1, \lambda)\right\}=\frac{E\left\{z^{\lambda}\right\}}{B(\lambda)} E\left\{x^{-v} H\right\}
$$

The expected rates of return in equations (35) and (36) are expectations based on the objective distributions of equilibrium rates of return. In the case in which subjective distributions differ from objective distributions, the subjective distributions guide behavior, and equilibrium rates of return depend on subjective distributions. However, in a long time series of observations on equilibrium rates of return, the sample average will correspond to the objective expectations of these rates of return (which depend on subjective distributions through $A(\lambda), B(\lambda)$, and $\Psi(\lambda)$ in equations (20), (21), and (31), respectively) as in equations (35) and (36). I will use these equations to calculate risk, term, and equity premia, which I will define in Section 3.

\section{Risk, Term, and Equity Premia}

I will define the risk premium for the set of canonical assets with a given value of $\lambda$ as the excess of the expected rate of return on the one-period version of the canonical asset with that value of $\lambda$ relative to the rate of return on a one-period riskless bill. Specifically, I will define the unconditional risk premium $R P(\lambda)$ as

$$
R P(\lambda) \equiv \frac{E\left\{R_{t+1}(1, \lambda)\right\}}{E\left\{R_{t+1}(1,0)\right\}}-1 .
$$

Use the expression for the expected rate of return on one-period canonical assets from equation (36) to rewrite the risk premium in equation (37) as

$$
R P(\lambda)=\frac{B(0)}{B(\lambda)} E\left\{z^{\lambda}\right\}-1
$$


Equation (38) can be rewritten by using the definition of $B(\lambda)$ in equation (21) to obtain

$$
R P(\lambda)=\frac{E^{*}\left\{x^{-\eta} H\right\} E\left\{z^{\lambda}\right\}}{E^{*}\left\{x^{-\eta} H z^{\lambda}\right\}}-1 .
$$

To interpret the sources of the risk premium in equation (39), rewrite this equation as

$$
1+R P(\lambda)=\frac{E^{*}\left\{x^{-\eta} H\right\} E^{*}\left\{z^{\lambda}\right\}}{E^{*}\left\{x^{-\eta} H z^{\lambda}\right\}} \times \frac{E\left\{z^{\lambda}\right\}}{E^{*}\left\{z^{\lambda}\right\}} .
$$

This risk premium-more precisely, $1+R P(\lambda)$-is the product of two ratios on the right hand side of equation (40). The first ratio differs from one to the extent that there is a nonzero subjective covariance between the risky payoff in period $t+1, z_{t+1}^{\lambda}$, and the part of the pricing kernel in equation (10) that is unpredictable one period in advance, $x_{t+1}^{-\eta} H_{t+1}$. If $z_{t+1}^{\lambda}$ is (subjectively) uncorrelated with $x_{t+1}^{-\eta} H_{t+1}$, this component of $1+R P(\lambda)$ equals one, and thus it does not contribute to the risk premium. If $z_{t+1}^{\lambda}$ is negatively correlated with $x_{t+1}^{-\eta} H_{t+1}$ under the subjective distribution, so that the risky payoff is high when the marginal utility of consumption is low, then the risk premium is positive. Under rational expectations, the second ratio on the right hand side of equation (40) equals one, and this term does not contribute to the risk premium. However, if the representative consumer is pessimistic, in the sense that the subjective expectation $E^{*}\left\{z^{\lambda}\right\}$ is less than the objective expectation $E\left\{z^{\lambda}\right\}$, this ratio exceeds one, which contributes to a positive risk premium. Pessimistic consumers will underprice the risky asset, leading to an increase in the realized rate of return on this asset. Equivalently, with $E\left\{z^{\lambda}\right\}>E^{*}\left\{z^{\lambda}\right\}$, the realized rate of return on the risky asset is higher than the representative consumer expects.

For any given value of $\lambda$, I will define the term premium as the excess of the expected rate of return on a security with maturity $n>1$ relative to the expected rate of return on a security with the same value of $\lambda$ and with a maturity of one period. Specifically, I will define the unconditional term premium $T P(n, \lambda)$ as

$$
T P(n, \lambda) \equiv \frac{E\left\{R_{t+1}(n, \lambda)\right\}}{E\left\{R_{t+1}(1, \lambda)\right\}}-1 .
$$

Substituting the expected rate of return on the canonical asset from equation (35) into the definition of the term premium in equation (41) and using 
equation (36) yields

$$
T P(n, \lambda)=A(\lambda)[\Psi(\lambda)-1] \frac{1-[A(\lambda)]^{n-1}}{1-[A(\lambda)]^{n}} .
$$

The sign of the term premium is the same as the sign of $\Psi(\lambda)-1$. The magnitude of the term premium is increasing in maturity $n$. For an infinitematurity asset, such as a stock or a consol, the term premium is computed by taking the limit as $n \rightarrow \infty$ to obtain

$$
T P(\infty, \lambda)=A(\lambda)[\Psi(\lambda)-1] .
$$

I will define the equity premium as the excess of the expected rate of return on the canonical asset with $n=\infty$ and $\lambda>0$ relative to the rate of return on a one-period riskless bill $(n=1, \lambda=0)$. Specifically, I will define the unconditional equity premium $E P(\lambda)$ as

$$
E P(\lambda) \equiv \frac{E\left\{R_{t+1}(\infty, \lambda)\right\}}{E\left\{R_{t+1}(1,0)\right\}}-1
$$

The definitions of the risk premium in equation (37) and the term premium in equation (41) imply that

$$
E P(\lambda)=(1+R P(\lambda))(1+T P(\infty, \lambda))-1 .
$$

Substitute the risk premium from equation (39) and the term premium from equation (43) into equation (45) to obtain

$$
E P(\lambda)=[1-A(\lambda)+\Psi(\lambda) A(\lambda)] \frac{E^{*}\left\{x^{-\eta} H\right\} E\left\{z^{\lambda}\right\}}{E^{*}\left\{x^{-\eta} H z^{\lambda}\right\}}-1 .
$$

I have defined the risk, term, and equity premia as ratios of unconditional expectations of rates of return on various assets. The values of these premia would be unchanged if they were defined as ratios of conditional expectations of the same rates of return. Specifically, equation (25) and the fact that $\left(x_{t}, z_{t}, H_{t}\right)$ is i.i.d. over time imply that for any two canonical assets characterized by $\left(n_{1}, \lambda_{1}\right)$ and $\left(n_{2}, \lambda_{2}\right)$, respectively,

$$
\frac{E_{t}\left\{R_{t+1}\left(n_{1}, \lambda_{1}\right)\right\}}{E_{t}\left\{R_{t+1}\left(n_{2}, \lambda_{2}\right)\right\}}=\frac{E_{t}\left\{J\left(x_{t+1}, z_{t+1}, H_{t+1} ; n_{1}, \lambda_{1}\right)\right\}}{E_{t}\left\{J\left(x_{t+1}, z_{t+1}, H_{t+1} ; n_{2}, \lambda_{2}\right)\right\}}=\frac{E\left\{R_{t+1}\left(n_{1}, \lambda_{1}\right)\right\}}{E\left\{R_{t+1}\left(n_{2}, \lambda_{2}\right)\right\}} .
$$

Thus, for instance, the conditional equity premium, $\frac{E_{t}\left\{R_{t+1}(\infty, \lambda)\right\}}{E_{t}\left\{R_{t+1}(1,0)\right\}}-1$, equals the unconditional equity premium defined in equation (44). Similarly, the conditional risk and term premia equal the respective unconditional risk and term premia defined in equations (37) and (41). 


\section{Lognormality}

Suppose that the objective distribution of the vector $(\ln x, \ln z)^{\prime}$ is $N(\mu, \Sigma)$ where $\mu=\left(\mu_{x}, \mu_{z}\right)^{\prime}$ and $\Sigma=\left(\begin{array}{cc}\sigma_{x}^{2} & \sigma_{x z} \\ \sigma_{x z} & \sigma_{z}^{2}\end{array}\right)$. It is straightforward to show that for constants $a, b$, and $\lambda$,

$$
\frac{E\left\{x^{a}\right\} E\left\{z^{\lambda}\right\}}{E\left\{x^{b} z^{\lambda}\right\}}=\exp \left[(a-b) \mu_{x}+0.5\left(a^{2}-b^{2}\right) \sigma_{x}^{2}-b \lambda \sigma_{x z}\right]
$$

and

$$
\frac{E\left\{x^{a} z^{\lambda}\right\} E\left\{x^{b} z^{\lambda}\right\}}{E\left\{x^{a+b} z^{\lambda}\right\} E\left\{z^{\lambda}\right\}}=\exp \left[-a b \sigma_{x}^{2}\right] .
$$

Suppose that the subjective distribution of the vector $(\ln x, \ln z)^{\prime}$ is $N\left(\mu^{*}, \Sigma^{*}\right)$ where $\mu^{*}=\left(\mu_{x}^{*}, \mu_{z}^{*}\right)^{\prime}$ and $\Sigma^{*}=\left(\begin{array}{cc}\sigma_{x}^{* 2} & \sigma_{x z}^{*} \\ \sigma_{x z}^{*} & \sigma_{z}^{* 2}\end{array}\right)$. Let $\theta_{x} \equiv \mu_{x}^{*}-\mu_{x}$ and $\theta_{z} \equiv \mu_{z}^{*}-\mu_{z}$, so $\theta \equiv\left(\begin{array}{ll}\theta_{x} & \theta_{z}\end{array}\right)^{\prime}$ is the amount by which the mean of the subjective distribution, $\mu^{*}$, exceeds the mean of the objective distribution, $\mu$. Let $\left(\begin{array}{ll}\Omega_{x x} & \Omega_{x z} \\ \Omega_{x z} & \Omega_{z z}\end{array}\right) \equiv \Omega \equiv \Sigma^{*}-\Sigma$ be the amount by which the variancecovariance matrix of the subjective distribution exceeds that of the objective distribution. It is straightforward to show that

$$
\frac{E^{*}\left\{x^{a} z^{\lambda}\right\}}{E\left\{x^{a} z^{\lambda}\right\}}=\exp \left[\left(\begin{array}{ll}
a & \lambda
\end{array}\right) \theta+0.5\left(\begin{array}{ll}
a & \lambda
\end{array}\right) \Omega\left(\begin{array}{c}
a \\
\lambda
\end{array}\right)\right] .
$$

I will use equations (48), (49), and (50) to derive simple expressions for the risk, term, and equity premia under lognormality.

\section{Risk, Term, and Equity Premia Under Log- normality with Consumption Externalities and without Habit Formation}

Equation (35) is an analytic expression for the unconditional objective expectation of the rate of return on the canonical asset for arbitrary $n$ and $\lambda$. Given the values of various moments of the objective and subjective distributions of the exogenous stochastic variables $x_{t}, z_{t}$, and $H_{t}$, it is a simple 
matter to compute $A(\lambda), B(\lambda)$, and $\Psi(\lambda)$ (from equations (20), (21), and (31), respectively) and substitute the values into equation (35) to obtain the unconditional objective expectation of the rate of return on the canonical asset. Similarly, it is a simple matter to compute the risk premium $R P(\lambda)$ in equation (39) and the term premium $T P(\infty, \lambda)$ in equation (43). In order to derive easily interpreted expressions for the expected rate of return, the risk premium, the term premium, and the equity premium expressed as functions of the parameters of the distributions of the exogenous stochastic variables, henceforth I will restrict attention to cases in which (1) the exogenous growth rates $x_{t}$ and $z_{t}$ are jointly lognormal (and, as before, i.i.d. over time) and (2) $D=0$, so that preferences do not depend on the individual's own lagged consumption, though, of course, they may depend on current or lagged aggregate consumption. With $D=0$, we have $H_{t} \equiv 1$.

To calculate the risk premium under lognormality with $D=0$, rearrange equation (39) and set $H \equiv 1$ to obtain

$$
R P(\lambda)=\frac{E\left\{x^{-\eta}\right\} E\left\{z^{\lambda}\right\}}{E\left\{x^{-\eta} z^{\lambda}\right\}} \frac{E^{*}\left\{x^{-\eta}\right\}}{E\left\{x^{-\eta}\right\}} \frac{E\left\{x^{-\eta} z^{\lambda}\right\}}{E^{*}\left\{x^{-\eta} z^{\lambda}\right\}}-1 .
$$

Now use equations (48) and (50) to calculate the ratios of moments in equation (51) to obtain

$$
R P(\lambda)=\exp \left[\eta \lambda \sigma_{x z}-\lambda \theta_{z}+\eta \lambda \Omega_{x z}-0.5 \lambda^{2} \Omega_{z z}\right]-1
$$

As a step toward calculating the term premium, I will calculate $\Psi(\lambda)$ under lognormality and the assumption that $D=0$. Substitute $A(\lambda)$ from equation (20) and $B(\lambda)$ from equation (21) into the expression for $\Psi(\lambda)$ in equation (31), and use the fact that $H_{t} \equiv 1$ when $D=0$ to obtain

$$
\Psi(\lambda)=\frac{E\left\{x^{v} z^{\lambda}\right\} E\left\{x^{-\eta} z^{\lambda}\right\}}{E\left\{z^{\lambda}\right\} E\left\{x^{v-\eta} z^{\lambda}\right\}} \times \frac{E\left\{x^{v-\eta} z^{\lambda}\right\}}{E^{*}\left\{x^{v-\eta} z^{\lambda}\right\}} \times \frac{E^{*}\left\{x^{-\eta} z^{\lambda}\right\}}{E\left\{x^{-\eta} z^{\lambda}\right\}} .
$$

The expression for $\Psi(\lambda)$ in equation (53) is the product of three factors. The first factor is a ratio in which the numerator is the product of two expectations and the denominator is the product of two expectations. If the variables $x$ and $z$ were deterministic, the numerator and the denominator of this ratio would be equal and this factor would equal one. The second factor is the ratio of the objective expectation of a random variable to the subjective expectation of the same variable. The third factor is the ratio 
of the subjective expectation of a different random variable to the objective expectation of that variable. Under rational expectations, in which the subjective expectations of variables equal the objective expectations of those variables, the second and third factors are both equal to one.

Under the assumption of lognormality, the first factor on the right hand side of equation (53) can be calculated using equation (49) and the second and third factors on the right hand side of equation (53) can be calculated using equation (50) to obtain

$$
\Psi(\lambda)=\exp \left[v \eta \sigma_{x}^{2}-v \theta_{x}+v[\eta-0.5 v] \Omega_{x x}-v \lambda \Omega_{x z}\right] .
$$

$\Psi(\lambda)$ is one component of the term premium in equation (43). The other component of the term premium is $A(\lambda)$, which is defined in equation (20). Under lognormality, $A(\lambda)$ is given by

$$
A(\lambda)=\beta \exp \left[(v-\eta) \mu_{x}^{*}+\lambda \mu_{z}^{*}+0.5\left(\begin{array}{ll}
v-\eta & \lambda
\end{array}\right) \Sigma^{*}\left(\begin{array}{c}
v-\eta \\
\lambda
\end{array}\right)\right] .
$$

Note that under lognormality with $D=0$, so that $H_{t} \equiv 1$, the definition of $B(\lambda)$ in equation (21) implies that

$$
B(\lambda)=\beta \exp \left[-\eta \mu_{x}^{*}+\lambda \mu_{z}^{*}+0.5\left(\eta^{2} \sigma_{x}^{* 2}+\lambda^{2} \sigma_{z}^{* 2}-2 \eta \lambda \sigma_{x z}^{*}\right)\right] .
$$

Substituting $\Psi(\lambda)$ from equation (54) into the expression for the term premium in equation (43) gives a closed-form expression for the term premium of an infinite-maturity canonical asset in terms of the parameters of the subjective and objective lognormal distributions,

$$
T P(\infty, \lambda)=A(\lambda)\left[\exp \left(v \eta \sigma_{x}^{2}-v \theta_{x}+v[\eta-0.5 v] \Omega_{x x}-v \lambda \Omega_{x z}\right)-1\right],
$$

where $A(\lambda)$ is given by equation (55). Under rational expectations, $\theta=0$ and $\Omega=0$, so the term premium is simply $T P(\infty, \lambda)=A(\lambda)\left[\exp \left(v \eta \sigma_{x}^{2}\right)-1\right]$, which has the same sign as $v \eta \sigma_{x}^{2}$. Since $\eta \sigma_{x}^{2}>0$, the term premium has the same sign as $v$. Under standard time-separable preferences, $\gamma=0$ so that $v=0$ and the term premium is zero. With a benchmark level of consumption, $\gamma>0$, so that $v$ and the term premium have the same sign as $\alpha-1$, which can be positive, zero, or negative.

To compute the equity premium under lognormality without habit formation, i.e., with $D=0$, set $H_{t} \equiv 1$ and apply equations (48) and (50) to 
equation (46) to obtain

$E P(\lambda)=[1-A(\lambda)+\Psi(\lambda) A(\lambda)] \exp \left[\eta \lambda \sigma_{x z}-\lambda \theta_{z}+\eta \lambda \Omega_{x z}-0.5 \lambda^{2} \Omega_{z z}\right]-1$,

where $A(\lambda)$ is given by equation (55) and $\Psi(\lambda)$ is given by equation (54).

\section{Linear Approximations to Risk, Term, and Equity Premia}

I have derived exact expressions for the risk premium, term premium, and equity premium under lognormality and $D=0$. These expressions are very easy to implement, but they are cumbersome to interpet. To understand the effects of various parameters on these premia, I will linearize the exact expressions as functions of the parameters. Specifically, I will treat each of these premia as functions of $\beta, \mu, \Sigma, \theta, \Omega$, and I will linearize each of these premia around $\beta=1, \mu=\theta=0$ and $\Sigma=\Omega=0$. Define $\phi \equiv$ $\left(\beta, \mu_{x}, \mu_{z}, \sigma_{x}^{2}, \sigma_{x z}, \sigma_{z}^{2}, \theta_{x}, \theta_{z}, \Omega_{x x}, \Omega_{x z}, \Omega_{z z}\right)$ as a vector containing the effective discount factor $\beta$, the parameters of the objective lognormal distribution, and the difference between the parameter values of the subjective and objective lognormal distributions. Let $\phi_{0}=(1,0, \ldots, 0)$ be the value of $\phi$ around which I linearize the risk premium, term premium, and equity premium. Equations (54), (55), and (56) imply that

$$
\Psi\left(\lambda ; \phi_{0}\right)=A\left(\lambda ; \phi_{0}\right)=B\left(\lambda ; \phi_{0}\right)=1 .
$$

Linearizing the risk premium in equation (52) yields

$$
R P(\lambda) \cong \eta \lambda \sigma_{x z}-\lambda \theta_{z}+\eta \lambda \Omega_{x z}-0.5 \lambda^{2} \Omega_{z z}
$$

Under rational expectations, $\theta=0$ and $\Omega=0$, and the linearized risk premium is simply $\eta \lambda \sigma_{x z}$, which is (up to a logarithmic approximation) the negative of the conditional covariance of the pricing kernel (in which $x_{t+1}^{-\eta}$ is unpredictable one period in advance) and the growth rate, $z_{t+1}^{\lambda}$, of the risky payoff.

I will indicate that the term premium on an infinite-maturity canonical asset depends on the parameter vector $\phi$ by rewriting equation (43) as

$$
T P(\infty, \lambda ; \phi)=(\Psi(\lambda ; \phi)-1) A(\lambda ; \phi)
$$


Recall from equation (59) that $\Psi\left(\lambda ; \phi_{0}\right)=A\left(\lambda ; \phi_{0}\right)=1$. Therefore, $T P\left(\infty, \lambda ; \phi_{0}\right)=0$ and the first-order linear approximation of the term premium is

$$
T P(\infty, \lambda ; \phi) \cong \frac{\partial \Psi\left(\lambda ; \phi_{0}\right)}{\partial \phi}\left(\phi-\phi_{0}\right)
$$

Linearizing the expression for $\Psi(\lambda)$ in equation (54) yields the following linear approximation to the term premium on an infinite-maturity asset

$$
T P(\infty, \lambda ; \phi) \cong v \eta \sigma_{x}^{2}-v \theta_{x}+v[\eta-0.5 v] \Omega_{x x}-v \lambda \Omega_{x z} .
$$

Under rational expectations, $\theta=0$ and $\Omega=0$, so the linearized term premium is simply $v \eta \sigma_{x}^{2}$.

Equation (45) implies that the linear approximation to the equity premium is the sum of the linear approximations to the risk premium and to the term premium on an infinite-maturity asset. Therefore, equations (60) and (63) imply

$$
\begin{aligned}
E P(\lambda) \cong & \eta \lambda \sigma_{x z}+v \eta \sigma_{x}^{2}-v \theta_{x}-\lambda \theta_{z} \\
& +v(\eta-0.5 v) \Omega_{x x}+(\eta-v) \lambda \Omega_{x z}-0.5 \lambda^{2} \Omega_{z z}
\end{aligned}
$$

Under rational expectations, $\theta=0$ and $\Omega=0$, so the linearized equity premium is simply $\eta \lambda \sigma_{x z}+v \eta \sigma_{x}^{2}$.

\section{Second Moments}

In this section I calculate the variances of the conditional and unconditional objective distributions of the rate of return on the canonical asset under lognormality and without habit formation, so that $D=0$. Since $H_{t} \equiv 1$ when $D=0$, equation (25) implies

$$
R_{t+1}(n, \lambda)=J\left(x_{t+1}, z_{t+1}, 1 ; n, \lambda\right) K\left(x_{t}, 1\right) .
$$

The objective variance of $R_{t+1}(n, \lambda)$ conditional on information available at date $t$ is

$$
\operatorname{Var}_{t}\left\{R_{t+1}(n, \lambda)\right\}=\left[K\left(x_{t}, 1\right)\right]^{2} \operatorname{Var}\left\{J\left(x_{t+1}, z_{t+1}, 1 ; n, \lambda\right)\right\} .
$$

The variance of $J\left(x_{t+1}, z_{t+1}, 1 ; n, \lambda\right)$ on the right hand side of equation (66) is an unconditional variance because $J\left(x_{t+1}, z_{t+1}, 1 ; n, \lambda\right)$ is i.i.d., so its conditional and unconditional variances are identical. 
I will confine attention to two special cases of equation (66), corresponding to riskless one-period bills and to equity. In the case of riskless one-period bills, $n=1$ and $\lambda=0$, so equation (32) reveals that $J\left(x_{t+1}, z_{t+1}, 1 ; 1,0\right)$ is constant. Thus the conditional variance of the riskless one-period return is zero. Indeed, this conditional variance must be zero in order for the bill to be (conditionally) riskless. In the case of equity, $n=\infty$ and the conditional variance of the rate of return depends on the (unconditional) variance of $J\left(x_{t+1}, z_{t+1}, 1 ; \infty, \lambda\right)$, since $H_{t} \equiv 1$ when there is no habit formation (i.e., when $D=0$ ). Setting $n=\infty$ and $H_{t}=1$ in equation (32) yields

$$
\begin{aligned}
& J\left(x_{t+1}, z_{t+1}, 1 ; \infty, \lambda\right) \\
& =\frac{E\left\{z^{\lambda}\right\}}{B(\lambda)}\left[(1-A(\lambda)) \frac{z_{t+1}^{\lambda}}{E\left\{z^{\lambda}\right\}}+\Psi(\lambda) A(\lambda) \frac{x_{t+1}^{v} z_{t+1}^{\lambda}}{E\left\{x^{v} z^{\lambda}\right\}}\right] .
\end{aligned}
$$

Therefore,

$$
E\left\{J\left(x_{t+1}, z_{t+1}, 1 ; \infty, \lambda\right)\right\}=\frac{E\left\{z^{\lambda}\right\}}{B(\lambda)}[1-A(\lambda)+\Psi(\lambda) A(\lambda)],
$$

which is consistent with equation (33) with $n=\infty$. Use the facts that

$$
\begin{gathered}
E\left\{\left(\frac{z^{\lambda}}{E\left\{z^{\lambda}\right\}}\right)^{2}\right\}=\exp \left[\lambda^{2} \sigma_{z}^{2}\right], \\
E\left\{\left(\frac{x^{v} z^{\lambda}}{E\left\{x^{v} z^{\lambda}\right\}}\right)^{2}\right\}=\exp \left[v^{2} \sigma_{x}^{2}+\lambda^{2} \sigma_{z}^{2}+2 v \lambda \sigma_{x z}\right],
\end{gathered}
$$

and

$$
E\left\{\left(\frac{z^{\lambda}}{E\left\{z^{\lambda}\right\}}\right)\left(\frac{x^{v} z^{\lambda}}{E\left\{x^{v} z^{\lambda}\right\}}\right)\right\}=\exp \left[\lambda^{2} \sigma_{z}^{2}+v \lambda \sigma_{x z}\right]
$$

along with equation (67) to obtain

$$
\begin{aligned}
& E\left\{\left[J\left(x_{t+1}, z_{t+1}, 1 ; \infty, \lambda\right)\right]^{2}\right\} \\
= & {\left[\frac{E\left\{z^{\lambda}\right\}}{B(\lambda)}\right]^{2}\left[\begin{array}{c}
(1-A(\lambda))^{2} \exp \left[\lambda^{2} \sigma_{z}^{2}\right] \\
+[\Psi(\lambda) A(\lambda)]^{2} \exp \left[\lambda^{2} \sigma_{z}^{2}+v^{2} \sigma_{x}^{2}+2 v \lambda \sigma_{x z}\right] \\
+2(1-A(\lambda)) \Psi(\lambda) A(\lambda) \exp \left[\lambda^{2} \sigma_{z}^{2}+v \lambda \sigma_{x z}\right]
\end{array}\right] . }
\end{aligned}
$$


Use equations (68) and (72) along with the fact that $\operatorname{Var}\{u\}=E\left\{u^{2}\right\}-$ $[E\{u\}]^{2}$ to obtain

$$
\operatorname{Var}\left\{J\left(x_{t+1}, z_{t+1}, 1 ; \infty, \lambda\right)\right\}=\left[\frac{E\left\{z^{\lambda}\right\}}{B(\lambda)}\right]^{2} V(\lambda)
$$

where

$$
\begin{aligned}
V(\lambda) \equiv & (1-A(\lambda))^{2}\left(\exp \left[\lambda^{2} \sigma_{z}^{2}\right]-1\right) \\
& +[\Psi(\lambda) A(\lambda)]^{2}\left(\exp \left[\lambda^{2} \sigma_{z}^{2}+v^{2} \sigma_{x}^{2}+2 v \lambda \sigma_{x z}\right]-1\right) \\
& +2(1-A(\lambda)) \Psi(\lambda) A(\lambda)\left(\exp \left[\lambda^{2} \sigma_{z}^{2}+v \lambda \sigma_{x z}\right]-1\right) .
\end{aligned}
$$

Therefore, equations (66) and (73) and the fact that $K\left(x_{t}, 1\right)=x_{t}^{-v}$ imply that the conditional variance of the rate of return on an infinite-maturity asset, such as equity, is

$$
\operatorname{Var}_{t}\left\{R_{t+1}(\infty, \lambda)\right\}=\left[\frac{E\left\{z^{\lambda}\right\}}{B(\lambda)} x_{t}^{-v}\right]^{2} V(\lambda) .
$$

In order to calculate the unconditional variance of the rate of return on the canonical asset in equation (25), I will use the following identity for independent random variables $K$ (which is abbreviated notation for $K\left(x_{t}, H_{t}\right)$ in equation (27)) and $J$ (which is abbreviated notation for $J\left(x_{t+1}, z_{t+1}, H_{t+1} ; n, \lambda\right)$ in equation (26))

$$
\operatorname{Var}\{K \times J\}=E\left\{K^{2}\right\} E\left\{J^{2}\right\}-[E\{K\}]^{2}[E\{J\}]^{2} .
$$

Rearrange equation (76) and use the fact that $R_{t+1}(\infty, \lambda)=K \times J$ to obtain $\operatorname{Var}\left\{R_{t+1}(\infty, \lambda)\right\}=\operatorname{Var}\{K\} \operatorname{Var}\{J\}+[E\{K\}]^{2} \operatorname{Var}\{J\}+[E\{J\}]^{2} \operatorname{Var}\{K\}$.

Equation (27) implies that when $D=0, K\left(x_{t}, H_{t}\right)=x_{t}^{-v}$, which is i.i.d. Under lognormality with $D=0$,

$$
E\left\{K\left(x_{t}, 1\right)\right\}=\exp \left[-v \mu_{x}+0.5 v^{2} \sigma_{x}^{2}\right]
$$

and

$$
\operatorname{Var}\left\{K\left(x_{t}, 1\right)\right\}=\left[\exp \left(v^{2} \sigma_{x}^{2}\right)-1\right] \exp \left[-2 v \mu_{x}+v^{2} \sigma_{x}^{2}\right]
$$


To summarize, the objective unconditional variance of the rate of return on equity under lognormality with $D=0$ is given by equation (77), where $E\{K\}$ is given by equation (78), $\operatorname{Var}\{K\}$ is given by equation (79), $E\{J\}$ is given by equation (68), and $\operatorname{Var}\{J\}$ is given by equation (73).

For riskless one-period bills $(n=1, \lambda=0)$, the unconditional variance is particularly simple. Equation (22) together with $a_{0}=1$ implies $\omega(1,0)=$ $B(0)$, so that equation (24) implies

$$
R_{t+1}(1,0)=\frac{1}{B(0)} x_{t}^{-v}
$$

when $D=0$, so that $H_{t} \equiv 1$. Therefore, the unconditional variance of the rate of return on the one-period riskless asset is

$$
\operatorname{Var}\left\{R_{t+1}(1,0)\right\}=[B(0)]^{-2}\left[\exp \left(v^{2} \sigma_{x}^{2}\right)-1\right] \exp \left[-2 v \mu_{x}+v^{2} \sigma_{x}^{2}\right] .
$$

\subsection{Linear Approximations to Second Moments}

I will linearize the conditional and unconditional variances of the rate of return on the canonical asset around the point $\phi=\phi_{0}$, where, as in Section $6, \phi \equiv\left(\beta, \mu_{x}, \mu_{z}, \sigma_{x}^{2}, \sigma_{x z}, \sigma_{z}^{2}, \theta_{x}, \theta_{z}, \Omega_{x x}, \Omega_{x z}, \Omega_{z z}\right)$ and $\phi_{0}=(1,0, \ldots, 0)$. For infinite-maturity assets, such as stocks or consols, the linear approximation to the conditional variance is obtained by first linearizing $\operatorname{Var}\left\{J\left(x_{t+1}, z_{t+1}, 1 ; \infty, \lambda\right)\right\}$ in equation (73), using equation (59), to obtain

$$
\operatorname{Var}\left\{J\left(x_{t+1}, z_{t+1}, 1 ; \infty, \lambda\right)\right\} \cong \widetilde{\operatorname{Var}}\{J\} \equiv v^{2} \sigma_{x}^{2}+\lambda^{2} \sigma_{z}^{2}+2 v \lambda \sigma_{x z} .
$$

Substituting equation (82) into equation (66), and using the fact that $K\left(x_{t}, 1\right)=$ $x_{t}^{-v}$ yields the linearized conditional variance

$$
\operatorname{Var}_{t}\left\{R_{t+1}(\infty, \lambda)\right\} \cong x_{t}^{-2 v}\left(v^{2} \sigma_{x}^{2}+\lambda^{2} \sigma_{z}^{2}+2 v \lambda \sigma_{x z}\right)
$$

As a step toward linearizing the unconditional variance of the return on an infinite-maturity canonical asset, observe from equation (78) that

$$
E\left\{K\left(x_{t}, 1 ; \phi_{0}\right)\right\}=1
$$

Using equation (59), equation (68) implies that

$$
E\left\{J\left(x_{t+1}, z_{t+1}, 1 ; \infty, \lambda ; \phi_{0}\right)\right\}=1 .
$$


Equations (73) and (79) imply that

$$
\operatorname{Var}\left\{J\left(x_{t+1}, z_{t+1}, 1 ; \infty, \lambda ; \phi_{0}\right)\right\}=\operatorname{Var}\left\{K\left(x_{t}, 1 ; \phi_{0}\right)\right\}=0 .
$$

Linearizing equation (77) around $\phi=\phi_{0}$, and using equations (84), (85), and (86) yields

$$
\operatorname{Var}\left\{R_{t+1}(\infty, \lambda)\right\} \cong \widetilde{\operatorname{Var}}\{J\}+\widetilde{\operatorname{Var}}\{K\},
$$

where $\widetilde{\operatorname{Var}}\{J\}$ is the linearized variance of $J\left(x_{t+1}, z_{t+1}, 1 ; \infty, \lambda\right)$ in equation (82), and $\widehat{\operatorname{Var}}\{K\} \equiv v^{2} \sigma_{x}^{2}$ is the linearized variance of $K\left(x_{t}, 1\right)$, obtained by linearizing equation (79). Therefore,

$$
\operatorname{Var}\left\{R_{t+1}(\infty, \lambda)\right\} \cong 2 v^{2} \sigma_{x}^{2}+\lambda^{2} \sigma_{z}^{2}+2 v \lambda \sigma_{x z} .
$$

The linearized unconditional variance of the one-period riskless rate is calculated by linearizing equation (81) to obtain

$$
\operatorname{Var}\left\{R_{t+1}(1,0)\right\} \cong v^{2} \sigma_{x}^{2} .
$$

Notice that the linearized variances (conditional and unconditional) of the riskless rate and the rate of return on equity depend on the moments of the objective distributions, but do not depend on any deviation of the moments of the subjective distributions from the corresponding moments of the objective distributions. Of course, the linearizations are expansions around $\phi=\phi_{0}$ where the means and second moments of the objective and subjective distributions are both equal to zero.

\section{Correlation of dividend-price ratio and the rate of return on stock}

Let $\varphi_{t}(\lambda) \equiv \frac{y_{t}^{\lambda}}{p_{t}(\infty, \lambda)}$ be the dividend-price ratio of an infinite-maturity asset, such as equity, for which $a_{j}=1$ for all $j$. Equation (18), the limit as $n \rightarrow \infty$ of equation (28), and the definition of $K\left(x_{t}, H_{t}\right)$ in equation (27) imply

$$
\varphi_{t}(\lambda)=\frac{1-A(\lambda)}{B(\lambda)} K\left(x_{t}, H_{t}\right) .
$$

Recall from equation (25) that, with $n=\infty$, the rate of return on stock is $R_{t+1}(\infty, \lambda)=J\left(x_{t+1}, z_{t+1}, H_{t+1} ; \infty, \lambda\right) K\left(x_{t}, H_{t}\right)$, where $J\left(x_{t+1}, z_{t+1}, H_{t+1} ; \infty, \lambda\right)$ 
and $K\left(x_{t}, H_{t}\right)$ are independent of each other. To the extent that $K\left(x_{t}, H_{t}\right)$ exhibits variation, the dividend-price ratio will vary, and this variation will help predict the rate of return on stock. To measure the correlation between the dividend-price ratio and the rate of return on stock, I will use the following lemma.

Lemma 1 Let $X$ and $Y$ be independent random variables and let $a \neq 0$ be an arbitrary constant. Let $\nu_{X} \equiv \frac{\operatorname{Var}\{X\}}{[E\{X\}]^{2}}$ be the square of the coefficient of variation of $X$ and let $\nu_{Y} \equiv \frac{\operatorname{Var}\{Y\}}{[E\{Y\}]^{2}}$ be the square of the coefficient of variation of $Y$. Then the square of the correlation of $a X$ and $X Y$ is $R^{2}=\frac{\nu_{X}}{\nu_{X} \nu_{Y}+\nu_{X}+\nu_{Y}}$.

Proof. $R^{2}=\frac{\left[E\left\{a X^{2} Y\right\}-E\{a X\} E\{X Y\}\right]^{2}}{\operatorname{Var}\{a X\} \operatorname{Var}\{X Y\}}$. Since $X$ and $Y$ are independent, $R^{2}=\frac{[E\{Y\}]^{2}[\operatorname{Var}\{X\}]^{2}}{\operatorname{Var}\{X\} \operatorname{Var}\{X Y\}}=\frac{[E\{Y\}]^{2} \operatorname{Var}\{X\}}{\operatorname{Var}\{X Y\}}$. Use the identity for independent variables in equation (77) to obtain an expression for $\operatorname{Var}\{X Y\}$, and rewrite $R^{2}$ as $R^{2}=\frac{[E\{Y\}]^{2} \operatorname{Var}\{X\}}{\operatorname{Var}\{X\} \operatorname{Var}\{Y\}+[E\{Y\}]^{2} \operatorname{Var}\{X\}+[E\{X\}]^{2} \operatorname{Var}\{Y\}}$. Divide the numerator and denominator by $[E\{X\}]^{2}[E\{Y\}]^{2}$ and use the definition of the coefficient of variation to obtain $R^{2}=\frac{\nu_{X}}{\nu_{X} \nu_{Y}+\nu_{X}+\nu_{Y}}$.

Let $\nu_{K}$ be the square of the coefficient of variation of $K\left(x_{t}, H_{t}\right)$ and $\nu_{J}$ be the square of the coefficient of variation of $J\left(x_{t+1}, z_{t+1}, H_{t+1} ; \infty, \lambda\right)$. Applying Lemma 1 with $X=K\left(x_{t}, H_{t}\right), Y=J\left(x_{t+1}, z_{t+1}, H_{t+1} ; \infty, \lambda\right)$, and $a=\frac{1-A(\lambda)}{B(\lambda)}$, so that $\varphi_{t}(\lambda)=a X$ and $R_{t+1}(\infty, \lambda)=X Y$, the $R^{2}$ of a regression of the rate of return on equity on the dividend-price ratio is

$$
R^{2}=\frac{\nu_{K}}{\nu_{K} \nu_{J}+\nu_{K}+\nu_{J}} \text {. }
$$

The expression for $R^{2}$ in equation (91) is an exact expression and holds for the case with habit formation (so that $H_{t}$ is endogenous) as well as for the case in which $H_{t} \equiv 1$. Now, as in Sections 5, 6, and 7, I will confine attention to the case with lognormality and without habit formation so that $H_{t} \equiv 1$.

When $H_{t} \equiv 1, K\left(x_{t}, H_{t}\right)=x_{t}^{-v}$, so that under lognormality, the squared coefficient of variation of $K\left(x_{t}, H_{t}\right)$ is

$$
\nu_{K}=\exp \left(v^{2} \sigma_{x}^{2}\right)-1
$$

Substituting equation (92) into equation (91) yields

$$
R^{2}=\frac{\exp \left(v^{2} \sigma_{x}^{2}\right)-1}{\left(\nu_{J}+1\right) \exp \left(v^{2} \sigma_{x}^{2}\right)-1} .
$$


In the case of standard preferences without a benchmark level of consumption, $\gamma=0$, which implies that $v=0$, so that $R^{2}$ in equation (93) is zero. That is, the dividend-price ratio has no predictive power for the risky rate of return in the case with standard preferences. However, the introduction of a benchmark level of consumption allows $v$ to differ from zero and thus enables the dividend-price ratio to predict the rate of return on stock.

Now use the expression for the mean of $J\left(x_{t+1}, z_{t+1}, 1 ; \infty, \lambda\right)$ in equation (68) and the variance of $J\left(x_{t+1}, z_{t+1}, 1 ; \infty, \lambda\right)$ in equations (73) and (74) to obtain

$$
\left(\nu_{J}+1\right) \exp \left(v^{2} \sigma_{x}^{2}\right)=\frac{\Upsilon(\lambda)}{[1-A(\lambda)+\Psi(\lambda) A(\lambda)]^{2}},
$$

where

$$
\Upsilon(\lambda) \equiv \begin{gathered}
(1-A(\lambda))^{2} \exp \left[v^{2} \sigma_{x}^{2}+\lambda^{2} \sigma_{z}^{2}\right] \\
+[\Psi(\lambda) A(\lambda)]^{2} \exp \left[\lambda^{2} \sigma_{z}^{2}+2 v^{2} \sigma_{x}^{2}+2 v \lambda \sigma_{x z}\right] \\
+2(1-A(\lambda)) \Psi(\lambda) A(\lambda) \exp \left[v^{2} \sigma_{x}^{2}+\lambda^{2} \sigma_{z}^{2}+v \lambda \sigma_{x z}\right]
\end{gathered} .
$$

Finally, substitute equation (95) into equation (93) and multiply the numerator and denominator of the resulting expression by $[1-A(\lambda)+\Psi(\lambda) A(\lambda)]^{2}$ to obtain

$$
R^{2}=\frac{[1-A(\lambda)+\Psi(\lambda) A(\lambda)]^{2}\left[\exp \left(v^{2} \sigma_{x}^{2}\right)-1\right]}{\Upsilon(\lambda)-[1-A(\lambda)+\Psi(\lambda) A(\lambda)]^{2}} .
$$

I will approximate $R^{2}$ around $\phi=\phi_{0}$ by taking the ratio of the linearized numerator of equation (96) to the linearized denominator of equation (96) to 
obtain $^{8}$

$$
R^{2} \cong \frac{v^{2} \sigma_{x}^{2}}{\lambda^{2} \sigma_{z}^{2}+2 v^{2} \sigma_{x}^{2}+2 v \lambda \sigma_{x z}} .
$$

As with the approximate variances of the riskless rate and the rate of return on equity, the approximate value of $R^{2}$ in equation (97) depends on the objective moments of the distribution of $\left(x_{t}, z_{t}\right)$ but does not depend on the subjective moments of the distribution of $\left(x_{t}, z_{t}\right)$.

\subsection{Correlation of dividend-price ratio and the excess rate of return on stock}

I have shown that the dividend-price ratio at time $t$ can be used to predict the rate of return on stock from period $t$ to period $t+1$. The ability to forecast the rate of return on stock in this model does not reflect any ability to forecast dividend growth because dividend growth, $z_{t+1}^{\lambda}$, is i.i.d. Instead, the predictability of the rate of return on stock is the direct result of the predictability of the intertemporal marginal rate of substitution in the case in which $v \neq 0$. The predictable component of the intertemporal marginal rate of substitution is captured by $K\left(x_{t}, H_{t}\right) \equiv x_{t}^{-v} H_{t}$. Since, as shown in equation (90), the dividend-price ratio is proportional to $K\left(x_{t}, H_{t}\right)$, and since, as shown in equation (25), the rate of return on the canonical asset is proportional to $K\left(x_{t}, H_{t}\right)$, variation in $K\left(x_{t}, H_{t}\right)$ allows the dividend-price ratio to predict the rate of return on any asset that is a special case of the

\footnotetext{
${ }^{8}$ Rewrite equation (96) as
}

$$
R^{2}=\frac{R_{N}^{2}}{R_{D}^{2}}
$$

where

$$
R_{N}^{2}=[1-A(\lambda)+\Psi(\lambda) A(\lambda)]^{2}\left[\exp \left(v^{2} \sigma_{x}^{2}\right)-1\right]
$$

is the numerator of $R^{2}$ and

$$
R_{D}^{2}=\Upsilon(\lambda)-[1-A(\lambda)+\Psi(\lambda) A(\lambda)]^{2}
$$

is the denominator of $R^{2}$. When $\phi=\phi_{0}, R_{N}^{2}=0$ and $R_{D}^{2}=0$. Linearizing $R_{N}^{2}$ around $\phi=\phi_{0}$ yields $R_{N}^{2} \cong v^{2} \sigma_{x}^{2}$ and linearizing $R_{D}^{2}$ around $\phi=\phi_{0}$ yields $R_{D}^{2} \cong$ $2 \widetilde{\Psi}+2 \widetilde{A}+\lambda^{2} \sigma_{z}^{2}+2 v^{2} \sigma_{x}^{2}+2 v \lambda \sigma_{x z}-2 \widetilde{A}-2(-\widetilde{A}+\widetilde{\Psi}+\widetilde{A})$ where $\widetilde{\Psi}$ is the linearized value of $\Psi(\lambda)$ and $\widetilde{A}$ is the linearized value of $A(\lambda)$. Therefore, $R_{D}^{2} \cong \lambda^{2} \sigma_{z}^{2}+2 v^{2} \sigma_{x}^{2}+2 v \lambda \sigma_{x z}$. Taking the ratio of the approximate value of $R_{N}^{2}$ to the approximate value of $R_{D}^{2}$ yields equation (97) in the text. 
canonical asset. For instance, the dividend-price ratio can predict the rate of return on stock and the rate of return on one-period riskless bills. However, the dividend-price ratio cannot predict the excess rate of return on stock relative to one-period riskless bills, when the excess rate of return is defined as the ratio of the gross rates of return on the two assets. Specifically, using equation (25), the excess rate of return on stocks, expressed in ratio form, is

$$
\frac{R_{t+1}(\infty, \lambda)}{R_{t+1}(1,0)}=\frac{J\left(x_{t+1}, z_{t+1}, H_{t+1} ; \infty, \lambda\right)}{J\left(x_{t+1}, z_{t+1}, H_{t+1} ; 1,0\right)},
$$

which is i.i.d., and hence is unpredictable. Indeed, the fact that the ratio of returns in equation (98) is i.i.d. accounts for the fact that conditional and unconditional premia are equal in this model.

An alternative formulation of the excess rate of return is the arithmetic difference between the rates of return on stocks and one-period riskless bills. Define $J_{t+1}^{S} \equiv J\left(x_{t+1}, z_{t+1}, H_{t+1} ; \infty, \lambda\right), J_{t+1}^{b} \equiv J\left(x_{t+1}, z_{t+1}, H_{t+1} ; 1,0\right)$, and $J_{t+1}^{E} \equiv J_{t+1}^{S}-J_{t+1}^{b}$, so that the rate of return on stock is $J_{t+1}^{S} \times K\left(x_{t}, H_{t}\right)$, the rate of return on one-period riskless bills is $J_{t+1}^{b} \times K\left(x_{t}, H_{t}\right)$, and the arithmetic excess rate of return on stocks is

$$
R_{t+1}(\infty, \lambda)-R_{t+1}(1,0)=J_{t+1}^{E} \times K\left(x_{t}, H_{t}\right) .
$$

The excess rate of return in equation (99) is proportional to $K\left(x_{t}, H_{t}\right)$ and thus is predictable by the dividend-price ratio, which is also proportional to $K\left(x_{t}, H_{t}\right)$. However, for empirically plausible values, the $R^{2}$ of the regression of the arithmetic excess return in equation (99) on the dividend-price ratio is extremely small. Applying equation (91), the $R^{2}$ of the regression of the arithmetic excess stock return on the dividend-price ratio is

$$
R^{2}=\frac{\nu_{K}}{\nu_{K} \nu_{J^{E}}+\nu_{K}+\nu_{J^{E}}},
$$

where is $\nu_{J^{E}}$ is the square of the coefficient of variation of $J_{t+1}^{E}$. Since $J_{t+1}^{b} \equiv$ $J\left(x_{t+1}, z_{t+1}, H_{t+1} ; 1,0\right)=\frac{a_{0}}{\omega(1,0)}$ is constant, $\operatorname{Var}\left\{J_{t+1}^{E}\right\}=\operatorname{Var}\left\{J_{t+1}^{S}\right\}$, so

$$
\nu_{J^{E}}=\frac{\operatorname{Var}\left\{J_{t+1}^{S}\right\}}{\left[E\left\{J_{t+1}^{S}\right\}-E\left\{J_{t+1}^{b}\right\}\right]^{2}} .
$$

Rearrange the right hand side of equation (101) using the facts that $E\left\{R_{t+1}(\infty, \lambda)\right\}=$ $E\left\{J_{t+1}^{S}\right\} E\left\{K\left(x_{t}, H_{t}\right)\right\}$ and $E\left\{R_{t+1}(1,0)\right\}=E\left\{J_{t+1}^{b}\right\} E\left\{K\left(x_{t}, H_{t}\right)\right\}$ to obtain

$$
\nu_{J^{E}}=\nu_{J^{S}} Q,
$$


where $Q \equiv\left[\frac{E\left\{R_{t+1}(\infty, \lambda)\right\}}{E\left\{R_{t+1}(\infty, \lambda)\right\}-E\left\{R_{t+1}(1,0)\right\}}\right]^{2}$ and $\nu_{J^{S}}$ is the squared coefficient of variation of $J_{t+1}^{S}$. Equations (100) and (102) and the fact that $\nu_{K} \nu_{J^{E}} \geq 0$ imply that

$$
R^{2} \leq \frac{\nu_{K}}{\nu_{K}+\nu_{J E}}=\frac{1}{1+\frac{\nu_{J} S}{\nu_{K}} Q} .
$$

To get a sense of the size of the upper bound on $R^{2}$ on the right hand side of equation (103), consider an average (gross) rate of return on stocks, $E\left\{R_{t+1}(\infty, \lambda)\right\}$, of 1.0698 per year and an average equity premium, $E\left\{R_{t+1}(\infty, \lambda)\right\}$ - $E\left\{R_{t+1}(1,0)\right\}$, of 0.0618 per year, as in Mehra and Prescott (1985). In this case, $Q=300$. Empirically, the ratio $\frac{\nu_{J} S}{\nu_{K}}$ is greater than one. If $\frac{\nu_{J} S}{\nu_{K}}$ were as low as one, the upper bound on $R^{2}$ would be $\frac{1}{1+300}=0.003$. In calibrations reported later in this chapter, $\frac{\nu_{J} S}{\nu_{K}}$ is greater than 5 , so the upper bound on $R^{2}$ is smaller than $\frac{1}{1+1500}=0.00067$.

\section{Special Cases}

In this section, I will examine the characteristics of rates of return in various special cases. I will adopt the normalization that the variance of $\ln z$ equals the variance of $\ln x$, i.e., that $\sigma_{z} \equiv \sigma_{x}$. With this normalization, $\lambda$ captures any difference in the standard deviations of the "un-normalized" values of $\ln z$ and $\ln x$. Also, with this normalization, the covariance $\sigma_{x z}=\rho \sigma_{x}^{2}$, where $-1 \leq \rho \leq 1$ is the correlation of $\ln x$ and $\ln z$.

\subsection{Rational Expectations}

Under rational expectations (RE), subjective distributions are identical to the corresponding objective distributions, so that $\theta=0$ and $\Omega=0$. In this case, the approximate equity premium in equation (64) simplifies to

$$
E P(\lambda) \cong(\lambda \rho+v) \eta \sigma_{x}^{2}, \quad \text { under RE, }
$$

which is the sum of the approximate risk premium from equation (60)

$$
R P(\lambda) \cong \lambda \rho \eta \sigma_{x}^{2}, \quad \text { under } \mathrm{RE},
$$

and the approximate term premium from equation (63)

$$
T P(\infty, \lambda) \cong v \eta \sigma_{x}^{2}, \quad \text { under RE. }
$$


The approximate unconditional variance of the rate of return on equity in equation (88) simplifies to

$$
\operatorname{Var}\left\{R_{t+1}(\infty, \lambda)\right\} \cong\left(2 v^{2}+\lambda^{2}+2 v \lambda \rho\right) \sigma_{x}^{2}, \quad \text { under RE. }
$$

The approximate unconditional variance of the one-period riskless rate under rational expectations is the same as in equation (89).

Finally, the approximate value of $R^{2}$ in equation (97) simplifies to

$$
R^{2} \cong \frac{v^{2}}{\lambda^{2}+2 v^{2}+2 v \lambda \rho}, \quad \text { under RE }
$$

The value of $R^{2}$ in equation (108) applies to a regression of the equity rate of return (rather than the excess equity rate of return) on the dividend-price ratio. As emphasized in subsection 8.1, excess returns are not predictable in the model presented here, so the model cannot account for the empirically observed predictability of excess returns. Nevertheless, the model can account for predictability of equity returns, and in the calculations throughout this section I will focus on the $R^{2}$ of the regressions of the equity rate of return on the dividend-price ratio.

\subsubsection{Perfectly Correlated $\ln x$ and $\ln z$}

In the original Lucas (1978) model of asset pricing in an exchange economy, equilibrium consumption per capita, $C_{t}$, is identically equal to the dividend per capita on risky stock, $y_{t}^{\lambda}$. In the current framework, the identity of consumption per capita and dividend per capita is represented by $C_{t} \equiv y_{t}$ and $\lambda=1$. Because $C_{t} \equiv y_{t}$, the growth rates of consumption and dividends are identically equal. That is, $x_{t} \equiv z_{t}$, so $\rho=1$. In this subsection, I will maintain the assumption that $\rho=1$, but I will allow $\lambda$ to differ from one to take account of levered equity.

In the remainder of this subsection on asset returns under rational expectations, I will examine values of the risk premium, term premium, equity premium, standard deviations of rates of return on one-period riskless bills and levered equity, and the $R^{2}$ of regressions of the equity rate of return on the dividend-price ratio. Table 2 presents the approximate values of these six features of asset returns for five particular cases. The final column of Table 2, labelled "Data" presents the empirical values of these features of the data. 


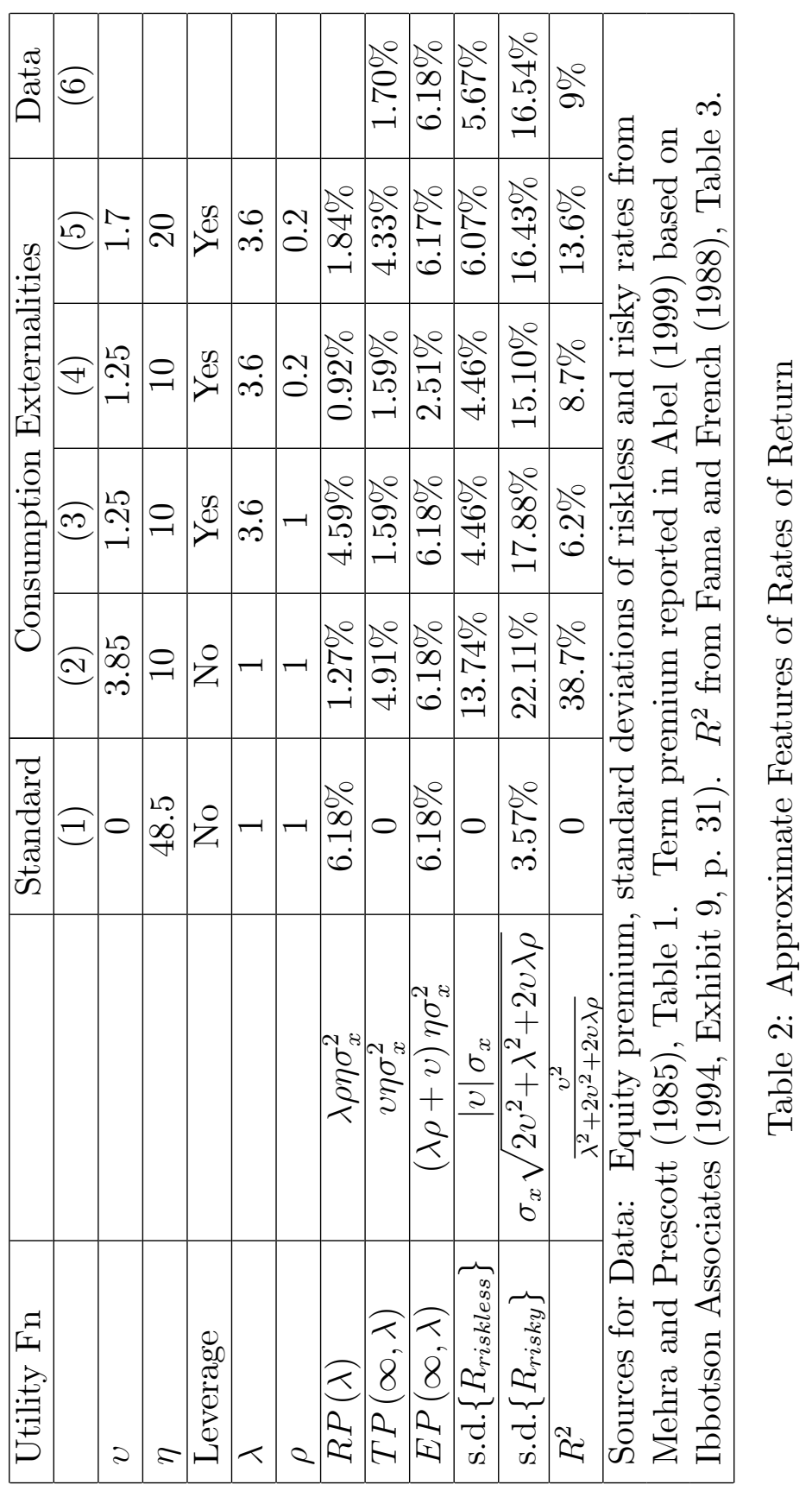


With $\rho=1$, the approximate equity premium in equation (104) simplifies to

$$
E P(\lambda) \cong(\lambda+v) \eta \sigma_{x}^{2}, \quad \text { under } \mathrm{RE}, \text { if } \rho=1,
$$

which is the sum of the approximate risk premium from equation (105)

$$
R P(\lambda) \cong \lambda \eta \sigma_{x}^{2}, \quad \text { under RE, if } \rho=1,
$$

and the approximate term premium from equation (106), which is invariant to $\rho$. The expression for the approximate unconditional variance of the rate of return on equity in equation (107) simplifies to

$$
\operatorname{Var}\left\{R_{t+1}(\infty, \lambda)\right\} \cong\left[(v+\lambda)^{2}+v^{2}\right] \sigma_{x}^{2}, \quad \text { under RE, if } \rho=1,
$$

and equation (108) implies that the $R^{2}$ of a regression of the rate of return on equity, $R_{t+1}(\infty, \lambda)$, on the dividend-price ratio, $\varphi_{t}(\lambda)$, is approximately

$$
R^{2} \cong \frac{v^{2}}{(\lambda+v)^{2}+v^{2}}, \quad \text { under RE, if } \rho=1 .
$$

Standard Utility Function and No Leverage With a standard utility function that does not include benchmark levels of consumption, $\gamma=0$, which implies $v=0$ and $\eta=\alpha$, where $\alpha$ is the coefficient of relative risk aversion. The seminal papers of Lucas (1978) and Mehra and Prescott (1985) analyze unlevered equity, which is represented here by the canonical asset with $n=\infty$ and $\lambda=1$. In this case, the approximate equity premium in equation (109) simplifies to

$$
E P(\lambda) \cong \alpha \sigma_{x}^{2}, \quad \text { under RE, if } \rho=1, \gamma=0, \text { and } \lambda=1 .
$$

In this case, since $v=0$, the term premium is zero, and the equity premium is composed entirely of the risk premium. Equation (113) is the essence of the Mehra-Prescott equity premium puzzle. Over the time period examined by Mehra and Prescott, 1889-1978, the average value of the equity premium was 0.0618 per year and the annual standard deviation of the growth rate of consumption per capita, measured here by $\sigma_{x}$, was 0.0357 . Thus, if the coefficient of relative risk aversion, $\alpha$, equals 10 , which is a very high value in the judgment of Mehra and Prescott, the equity premium would be only $(10)(0.0357)^{2}=0.0127$, i.e., $1.27 \%$ per year. In order for the model to match the historical equity premium of 0.0618 , the coefficient of relative risk 
aversion, $\alpha$, would need to equal $0.0618 /(0.0357)^{2}=48.5$, which is far higher than the upper bound of 10 imposed by Mehra and Prescott on the value of $\alpha .^{9}$

Note from equation (112) that with standard preferences, which imply $v=0$, the approximate value of $R^{2}$ is zero. Indeed, when $v=0$, the exact expression for $R^{2}$ in equation (96) is also equal to zero. Therefore, the dividend-price ratio cannot help predict the rate of return on equity in a linear regression. In addition, with $v=0$, the rate of return on oneperiod riskless bills is constant, which is indicated by a value of zero for s.d. $\left\{R_{\text {riskless }}\right\}$ in column (1) of Table 2 .

Consumption Externalities and No Leverage Consumption externalities, by allowing $v$ to be positive, can increase the equity premium associated with any given value of $\eta$, introduce variability in the one-period riskless rate of return and a positive term premium, and help account for the ability of the dividend-price ratio to predict equity returns, though as discussed in subsection 8.1, they cannot account for the predictability of excess returns.

In the absence of leverage, $\lambda=1$. Therefore, in this case, the approximate equity premium in equation (109) becomes

$$
E P(\lambda) \cong(1+v) \eta \sigma_{x}^{2}, \quad \text { under RE, if } \rho=1 \text { and } \lambda=1 .
$$

Thus, for given values of $\eta$ and $\sigma_{x}^{2}$, the equity premium implied by the model increases by a factor $1+v$. Therefore, the introduction of a positive $v$ allows the model to match the historical value of the equity premium with a smaller value of $\eta$. For instance, if $\eta=10$ (which would arise if, for instance, $\alpha=10$ and $\left.\theta_{0}=0\right)$, the model will deliver an approximate equity premium equal to its historical average value if $(1+v)=\frac{E P(\lambda)}{10 \sigma_{x}^{2}}=0.0618 /\left[10(0.0357)^{2}\right]$ $=4.85$, or equivalently, if $v=3.85$, as in column (2) of Table 2. In Abel (1990), I suggested that this formulation of utility might help account for the historically observed equity premium, but I acknowledged that the implied variability of the riskless rate was far larger than the historical variability of the riskless rate. Equation (89) implies that the approximate standard deviation of the one-period riskless rate is $|v| \sigma_{x}$, which equals $(3.85)(0.0357)$ $=13.74 \%$. However, the historical standard deviation of the riskless rate reported by Mehra and Prescott (1985) is only 5.67\% per year.

\footnotetext{
${ }^{9}$ Kandel and Stambaugh (1991) provide a compelling challenge to arguments that the coefficient of relative risk aversion is no larger than 10 .
} 
The fact that $v$ can differ from zero when utility depends on a benchmark level of consumption implies that benchmark utility introduces a term premium and also allows the dividend-price ratio to help predict the rate of return on equity. Substituting $v=3.85$ into equation (106) yields a term premium of $(3.85)(10)(0.0357)^{2}=0.0491$, or $4.91 \%$, which is much higher than the empirical value. For instance, in Abel (1999) I report, based on data from Ibbotson Associates (1994, Exhibit 9, p. 31), that over the period 1926-1993 the term premium on long-term government bonds relative to short-term riskless bills was about 170 basis points per year, or 0.0170. The term premium in the model is for infinite-maturity assets with risky payoffs, whereas the data apply to finite-maturity riskless claims. However, I have argued in Abel (1999), that these differences in maturity and risk may increase the term premium in the model, but by only a small fraction of one percent per year. Thus, the 491 basis point term premium produced by the model in this case is too high compared to the empirical term premium.

Finally, substituting $v=3.85$ into equation (112), with $\lambda=1$, shows that a linear regression of the rate of return on equity on the dividend-price ratio has an approximate $R^{2}$ of 0.387 , which is much higher than typical values of $R^{2}$ in such regressions. For instance, using annual real returns on the value-weighted NYSE portfolio, Fama and French (1988, Table 3) report $R^{2}=0.09$ for the period 1941-1986.

Thus, consumption externalities without leverage can account for the observed equity premium with a value of $\eta=10$. However, the implied value of $v, 3.85$, leads to too much variability in the one-period riskless rate of return, to too high a term premium, and to too high a value of $R^{2}$ in a regression of the equity rate of return on the dividend-price ratio. The next challenge is to account for the observed value of the equity premium with a lower value of $v$.

Consumption Externalities with Leverage Adding leverage to the model with consumption externalities allows the model to match the historical equity premium with a lower value of $v$. The lower value of $v$ reduces the variability of the riskless rate, the term premium, and the value of $R^{2}$ in the model toward more realistic values.

The standard deviation of the growth rate of dividends, $z_{t+1}^{\lambda}$, is approximately $\lambda$ times the standard deviation of $z_{t+1}$. Since $z_{t+1} \equiv x_{t+1}$, the standard deviation of the growth rate of dividends is approximately $\lambda$ times 
$\sigma_{x}$, the standard deviation of the growth rate of consumption. Using data from Table 2, p. 402, of Cecchetti, Lam, and Mark (1990), the standard deviation of dividend growth is 3.6 times the standard deviation of consumption growth, so I will set $\lambda=3.6$. With $\lambda=3.6$, the approximate equity premium in equation (109) will equal the historical value of 0.0618 with $\eta=10$ and $\sigma_{x}=0.0357$, if $v=0.0618 /\left(10(0.0357)^{2}\right)-3.6=1.25$. Because the introduction of leverage permits a reduction in the value of $v$, it also reduces the variability of the one-period riskless rate, the term premium, and the value of $R^{2}$. As shown in column (3) of Table 2, with $v=1.25$, the approximate standard deviation of the one-period riskless rate in equation (89) is $(1.25)(0.0357)=0.0446$, which is much closer to its historical value of $5.67 \%$, as reported by Mehra and Prescott (1985). Also, with $v=1.25$, the approximate term premium is $(1.25)(10)(0.0357)^{2}=0.0159$, which is much closer to the empirical value of the term premium of 0.0170 mentioned earlier. With $\lambda=3.6$ and $v=1.25$ equation (112) implies that the $R^{2}$ of a regression of the rate of return on equity on the dividend-price ratio is approximately 0.062, which is more in line with the values reported by Fama and French (1988). Finally, from equation (111), the approximate unconditional standard deviation of the rate of return on equity when $\lambda=3.6$ and $v=1.25$ is 0.1788 , which is not far from the value of $16.54 \%$ reported by Mehra and Prescott (1985).

\subsubsection{Imperfectly Correlated $\ln x$ and $\ln z$}

I have shown that with $\eta=10, v=1.25$, and $\lambda=3.6$, the rational expectations solution of the model with $\rho=1$ generates realistic values for five features of returns: the equity premium, the term premium, the unconditional standard deviation of the risky rate of return, the unconditional standard deviation of the riskless rate of return, and the $R^{2}$ of the regression of the equity rate of return on the dividend-price ratio. However, with $\rho=1$, consumption growth and dividend growth are perfectly correlated. I will show that reducing the value of $\rho$ to a more realistic value reduces the ability of the model to match the equity premium, unless the value of $\eta$ is allowed to be greater than 10 .

Campbell and Cochrane (1999) argue that the correlation between consumption growth and dividend growth is difficult to estimate accurately and ultimately settle on a value of 0.2 to use in their calculations. I will follow their lead and set $\rho=0.2$. Since the equity premium under rational 
expectations approximately equals $(\lambda \rho+v) \eta \sigma_{x}^{2}$, reducing the correlation $\rho$ to 0.2 from 1.0 substantially reduces the equity premium when $\lambda=3.6$ and $v=1.25$. In fact, when these values of $\rho, \lambda$, and $v$ are combined with $\eta=10$ and $\sigma_{x}=0.0357$, the approximate equity premium is only 0.0251 , which is less than half of the historical average equity premium. As shown in column (4) of Table 2, the other four features of the returns implied by these parameter values are not as far from empirical values. Specifically, the approximate standard deviation of the rate of return on stocks is 0.1510 , and the approximate $R^{2}$ of the regression of the equity rate of return on the dividend-price ratio is 0.087 . The approximate term premium and the approximate standard deviation of the riskless rate are invariant to the correlation $\rho$, and hence these features are the same in column (4) as in column $(3)$.

Doubling the value of the curvature parameter $\eta$ to 20 and increasing the value of $v$ slightly to 1.7 , while keeping $\lambda=3.6, \rho=0.2$ and $\sigma_{x}=0.0357$, allows the model to match the empirical value of the equity premium and produces standard deviations of the riskless rate and the rate of return on equity that are close to their empirical values. Specifically, as shown in column (5) of Table 2, these parameter values lead to an equity premium of 0.0617, a standard deviation of the riskless rate of 0.0607 , and a standard deviation of the rate of return on equity of 0.1643 . However, these parameter values lead to a value of the term premium of 0.0433 , which is too high compared to the data, and an $R^{2}$ of 0.136 , which is a bit high.

\subsection{Distorted Beliefs}

In order to focus on the role of distorted beliefs on rates of return, I will restrict attention to the case in which $x_{t} \equiv z_{t}$, so that $\sigma_{x}^{2}=\sigma_{x z}=\sigma_{z}^{2}$. I assume that the subjective distributions take account of the fact that $x_{t} \equiv z_{t}$, so that $\theta_{x}=\theta_{z}$ and $\Omega_{x x}=\Omega_{x z}=\Omega_{z z}$. In this case, the approximate equity premium in equation (64) simplifies to

$$
E P(\lambda) \cong(v+\lambda)\left(\eta \sigma_{x}^{2}-\theta_{x}+[\eta-0.5(v+\lambda)] \Omega_{x x}\right), \quad \text { if } x_{t} \equiv z_{t} .
$$

Note that $v+\lambda>0$ because $v>-1$ (equation (8)) and $\lambda \geq 1$. Although the distortion of beliefs, represented here by nonzero values of $\theta_{x}$ and $\Omega_{x x}$, affects the approximate equity premium, equations (89), (88), and (97) show, as mentioned earlier, that the distortion of beliefs does not affect the approximate unconditional variances of the riskless and risky rates of return, nor 
does it affect the approximate $R^{2}$ of a regression of the risky rate of return on the dividend-price ratio.

\subsubsection{Standard Utility Function and No Leverage}

In the case with standard utility $(\gamma=0$, so $v=0)$ and no leverage $(\lambda=1)$, the approximate equity premium in equation (115) simplifies to

$$
E P(1) \cong \eta\left(\sigma_{x}^{2}+\Omega_{x x}\right)-\left(\theta_{x}+0.5 \Omega_{x x}\right), \quad \text { if } x_{t} \equiv z_{t}, \gamma=0, \text { and } \lambda=1 .
$$

In Abel (2002), I have used the term pessimism to describe a situation in which the subjective distribution of the growth rate is first-order stochastically dominated by the objective distribution of the growth rate. When the growth rate is lognormal, I measure the amount of pessimism about the growth rate by $\Delta_{1}$, where $\Delta_{1}$ satisfies $E^{*}\{x\}=e^{-\Delta_{1}} E\{x\}$, which implies

$$
\Delta_{1}=-\left(\theta_{x}+0.5 \Omega_{x x}\right) .
$$

Substituting equation (117) into equation (116) yields

$$
E P(1) \cong \eta\left(\sigma_{x}^{2}+\Omega_{x x}\right)+\Delta_{1}, \quad \text { if } x_{t} \equiv z_{t}, \gamma=0, \text { and } \lambda=1 .
$$

It is evident from equation (118) that pessimism, measured by $\Delta_{1}$, increases the equity premium. The economic reason for this effect is that pessimism reduces the price of the risky asset, which increases the rate of return to anyone who buys this asset. On average, the realized rate of return is higher than the amount required by consumers to compensate them for the risk of holding equity because the realized rate of return on equity is higher, on average, than consumers expect. Indeed, in the special case with standard utility $(\gamma=0)$ and no leverage $(\lambda=0)$, an increase in pessimism about the expected growth rate causes an equal-sized increase in the objective average equity premium.

In Abel (2002), I have used the term doubt to describe a situation in which the subjective distribution of the growth rate is a mean-preserving spread of the objective distribution of the growth rate. When the growth rate is lognormal, an increase in doubt is represented by an increase in $\Omega_{x x} \equiv$ $\sigma_{x}^{* 2}-\sigma_{x}^{2}$, holding constant $\theta_{x}+0.5 \Omega_{x x}=-\Delta_{1}$. Equation (118) shows that an increase in doubt increases the equity premium. The economic reason is that an increase in doubt increases the perceived riskiness of equity and thus increases the equity premium that consumers require in order to hold equity. 


\subsubsection{Consumption Externalities with Leverage}

In the presence of consumption externalities and/or leverage, we can interpret the effects on the equity premium of distorted beliefs by generalizing the measure of pessimism. Instead of comparing subjective and objective distributions of the growth rate $x_{t+1}$, I will compare the subjective and objective

distributions of $x_{t+1}^{v+\lambda}$ (which, in the special case with standard utility $(v=0)$ and no leverage $(\lambda=1)$, is simply the growth rate $\left.x_{t+1}\right)$. Now when the growth rate is lognormal, I will measure the amount of pessimism about $x_{t+1}^{v+\lambda}$ by $\Delta_{v+\lambda}$, where $\Delta_{v+\lambda}$ satisfies $E^{*}\left\{x^{v+\lambda}\right\}=e^{-\Delta_{v+\lambda}} E\left\{x^{v+\lambda}\right\}$, which implies

$$
\Delta_{v+\lambda}=-(v+\lambda) \theta_{x}-0.5(v+\lambda)^{2} \Omega_{x x} .
$$

Substituting equation (119) into equation (115) yields

$$
E P(\lambda) \cong(v+\lambda) \eta\left(\sigma_{x}^{2}+\Omega_{x x}\right)+\Delta_{v+\lambda}, \quad \text { if } x_{t} \equiv z_{t},
$$

which has the same form and interpretation as equation (118). Again, an increase in pessimism $\Delta_{v+\lambda}$ increases the equity premium, and an increase in doubt, represented as an increase in $\Omega_{x x}$ holding $\Delta_{v+\lambda}$ constant, increases the equity premium.

\section{Accuracy of Approximations}

I have conducted the analysis in two distinct parts. First, I set up the model and derived exact solutions for the equilibrium price and expected rate of return on the canonical asset. Because the canonical asset is sufficiently general, I used it to calculate rates of return on various assets, including one-period riskless bills and infinite-horizon levered equity. To obtain closed-form expressions for these exact solutions, I restricted attention to the case without habit formation (but with consumption externalities including keeping/catching up with the Joneses) and with lognormally distributed growth rates of consumption and dividends. The second part of the analysis started with the exact solutions derived in the first part, and derived linear approximations to various moments of asset returns. The rationale for approximating these expressions was to help interpret closed-form solutions, in particular, to understand the effects of various parameters on the means and variances of returns. In Section 9.1, I examined some quantitative features of the model under rational expectations. For clarity, I used the 
approximate expressions for the moments of asset returns. In this section, I will briefly explore the accuracy of these approximations by comparing the values obtained by evaluating the approximate expressions with the values obtained by evaluating the corresponding exact expressions.

Before comparing the approximate and exact solutions, I need to address an issue regarding the parameter values. As discussed in Section 1, the primitive specification of preferences includes seven parameters: the curvature paramater $\alpha$, the rate of time preference $\delta$, the importance of the consumption-benchmark ratio as measured by $\gamma$, the relative weight $D$ of the consumer's own past consumption in the benchmark, the weights $\theta_{0}$ and $\theta_{1}$ on contemporaneous and lagged aggregate consumption in the benchmark, and the exogenous growth rate $G$ in the benchmark. For the purposes of asset pricing in the absence of habit formation (i.e., with $D=0$ ), these seven preference parameters can be summarized by the three parameters $\beta, \eta$, and $v$ defined in equations (6), (7), and (8), respectively. The approximate expressions for the equity premium, the term premium, the variances of the riskless rate of return and the rate of return on stocks, and the value of $R^{2}$ are invariant to $\beta$. Thus in Section $9.1 \mathrm{I}$ did not specify a value for $\beta$. However, the exact expressions for these features of asset returns depend on $\beta$. Thus, to compare the exact and approximate expressions, I need to specify a value for $\beta$.

I will choose a value for $\beta$ by calibrating the model to the exact expression for the riskless rate of return under rational expectations. In the case without habit formation $D=0$, which implies $H_{t} \equiv 1$, so setting $\lambda=0$ in equation (36) implies that the average one-period riskless rate is

$$
E\left\{R_{t+1}(1,0)\right\}=\frac{E\left\{x^{-v}\right\}}{B(0)} .
$$

Setting $\lambda=0$ in equation (21) to obtain $B(0)$, imposing rational expectations, and substituting the result into equation (121) yields

$$
E\left\{R_{t+1}(1,0)\right\}=\frac{E\left\{x^{-v}\right\}}{\beta E\left\{x^{-\eta}\right\}}
$$

Let $\overline{R^{f}}$ be the empirical average value of the one-period riskless rate. The model will yield an expected one-period riskless rate equal to $\overline{R^{f}}$ if $\beta$ is chosen to set the right hand side of equation (122) equal to $\overline{R^{f}}$. Under 
lognormality, the value of the $\beta$ that will allow the model to match this value of the expected riskless rate is

$$
\beta=\frac{\exp \left[(\eta-v) \mu_{x}+0.5\left(v^{2}-\eta^{2}\right) \sigma_{x}^{2}\right]}{\overline{R^{f}}} .
$$

In the case with consumption externalities and leverage under rational expectations with perfectly correlated consumption growth and dividend growth in Section 9.1.1, I have set $\mu_{x}=0.018, \sigma_{x}=0.0357, \eta=10$, and $v=1.25$. Mehra and Prescott (1985) report $\overline{R^{f}}=1.0080$. Substituting these values into equation (123) yields $\beta=1.09$. At first blush, a value of $\beta$ greater than one might lead to two concerns. One concern is that $\beta>1$ might imply that the rate of time preference $\delta$ is negative. However, the definition of $\beta$ in equation (6) shows that $\beta$ can exceed one with a positive rate of time preference $\delta$, if $G>1$ and $(1-D) \gamma(\alpha-1)>0$. The second concern is that some important infinite sums will fail to converge. However, as shown by Mehra (1988, Appendix) and Kocherlakota (1990), $\beta$ can be slightly greater than one in a growing economy without causing this problem. However, for the particular parameter values in this example, including $\lambda=3.6$, the value of $A(\lambda)$ exceeds one when $\beta=1.09$. In order to make $A(\lambda)$ less than one, so that the difference equation in equation (19) will converge, I will set $\beta=1.075 .^{10}$ Of course, reducing the value of $\beta$ is equivalent to increasing the rate of time preference, and as a result of the increased rate of time preference, the (net) expected riskless rate increases to 0.0227 , or $2.27 \%$ per year. Having chosen a value for $\beta$, I can compute the exact as well as the approximate values of the equity premium, the standard deviations of the riskless rate of return and the rate of return on stock, and the value of $R^{2}$ in a regression of stock returns on the dividend-price ratio. As illustrated in column (1) of Table 3, the approximations are quite close to the exact values for the equity premium, the standard deviation of the riskless rate, and the value of $R^{2}$. The approximation for the standard deviation of the risky rate of return is not as good, but is within $10 \%$ of the value obtained from the exact expression.

\footnotetext{
${ }^{10}$ The values of derived preference parameters $\beta, \eta$, and $v$ are consistent with various combinations of the seven fundamental preference parameters. If, for example, $\delta=0.01$, $G=1.01279, D=0, \alpha=14, \gamma=0.4979, \theta_{0}=0.618$, and $\theta_{1}=0.193$, then the values of the three derived parameters are $\beta=1.075, \eta=10$, and $v=1.25$, as in column (1) in Table 3. If, for example, $\delta=0.01, G=1.0076, D=0, \alpha=25, \gamma=0.3759, \theta_{0}=0.554$,
} 


\begin{tabular}{|c|c|c|c|c|}
\hline \multicolumn{5}{|c|}{ Exact and Approximate Moments of Returns } \\
\hline & \multicolumn{2}{|c|}{$(1)$} & \multicolumn{2}{|c|}{$(2)$} \\
\hline \multicolumn{5}{|l|}{ Preference Parameters } \\
\hline$\beta$ & \multicolumn{2}{|c|}{1.075} & \multicolumn{2}{|c|}{1.060} \\
\hline$v$ & \multicolumn{2}{|c|}{1.25} & \multicolumn{2}{|c|}{1.70} \\
\hline$\eta$ & \multicolumn{2}{|c|}{10} & \multicolumn{2}{|c|}{20} \\
\hline Leverage Parameter, $\lambda$ & \multicolumn{2}{|c|}{3.6} & \multicolumn{2}{|c|}{3.6} \\
\hline \multicolumn{5}{|l|}{ Growth Rate Parameters } \\
\hline$\mu_{x}=\mu_{z}$ & \multicolumn{2}{|c|}{0.018} & \multicolumn{2}{|c|}{0.018} \\
\hline$\sigma_{x}=\sigma_{z}$ & \multicolumn{2}{|c|}{0.0357} & \multicolumn{2}{|c|}{0.0357} \\
\hline$\rho$ & \multicolumn{2}{|c|}{1.0} & \multicolumn{2}{|c|}{0.2} \\
\hline Moments of Returns from Model & exact & approx. & exact & approx. \\
\hline Equity Premium & $6.37 \%$ & $6.18 \%$ & $6.36 \%$ & $6.17 \%$ \\
\hline s.d. $\left\{R_{\text {riskless }}\right\}$ & $4.57 \%$ & $4.46 \%$ & $6.19 \%$ & $6.07 \%$ \\
\hline s.d. $\left\{R_{\text {risky }}\right\}$ & $19.59 \%$ & $17.88 \%$ & $17.91 \%$ & $16.43 \%$ \\
\hline$R^{2}$ & $6.1 \%$ & $6.2 \%$ & $13.5 \%$ & $13.6 \%$ \\
\hline Expected (net) riskless rate & $2.27 \%$ & & $1.82 \%$ & \\
\hline
\end{tabular}

Table 3: Exact and Approximate Moments of Returns 
Table 3 also reports approximate and exact values for the case with consumption externalities and leverage under rational expectations with less than perfectly correlated consumption growth and dividend growth. As in Section 9.1.2, I have set the correlation $\rho=0.2$. As I discussed earlier, the model is better able to deliver empirically realistic values if $\eta$ is increased to 20 and if $v$ is increased to 1.7. In order for $A(\lambda)$ to be smaller than one, as required for the difference equation in equation (19) to converge, I have reduced $\beta$ to 1.060. Despite this reduction in $\beta$, relative to its value in column (1), the expected riskless rate is only $1.82 \%$ per year, which is lower than its value in column (1). Again, the approximate values of the equity premium, the standard deviation of riskless rate, and the value of $R^{2}$ are very close to the values obtained from the exact expressions. And the approximate value of the standard deviation of the risky rate is within $10 \%$ of the value obtained from the exact expression.

\section{Summary}

I have derived exact expressions for the price and rate of return on a canonical asset in a framework that extends the standard utility function to include habit formation, keeping/catching up with the Joneses, and possible departures from rational expectations. The canonical asset is general enough to include one-period assets and infinite-maturity levered equity, and thus can be used to calculate risk premia, term premia, and equity premia. To calculate exact closed-form expressions for the first and second moments of returns, I have restricted attention to the case that excludes habit formation, but includes keeping/catching up with the Joneses, and I have assumed that the growth rates of consumption and dividends are jointly i.i.d. lognormal. After deriving the exact expressions for the first and second moments of returns, I have derived linear approximations to these expressions to show clearly how these moments of returns are affected by various parameters. I have used these linear approximations to show, quantitatively, how well the model can account for the empirical values of the equity premium, the term premium, the standard deviations of the riskless return and the rate of return on levered equity, and the value of $R^{2}$ of a regression of stock returns on the dividend-price ratio. Finally, I showed that the approximations yield values

and $\theta_{1}=0.188$, then the values of the three derived parameters are $\beta=1.060, \eta=20$, and $v=1.70$, as in column (2) of Table 3 . 
of these moments that are close to those obtained from the exact solutions, for empirically relevant parameter values. 


\section{References}

[1] Abel, Andrew B., "Asset Prices under Habit Formation and Catching up with the Joneses," American Economic Review, 80, 2 (May 1990), 38-42.

[2] Abel, Andrew B., "Risk Premia and Term Premia in General Equilibrium," Journal of Monetary Economics, 43, (February 1999), 3-33.

[3] Abel, Andrew B., "An Exploration of the Effects of Pessimism and Doubt on Asset Returns," Journal of Economic Dynamics and Control," 26, 7-8 (July 2002), 1075-1092.

[4] Abel, Andrew B., "Optimal Taxation When Consumers Have Endogenous Benchmark Levels of Consumption," The Review of Economic Studies, 72, 1 (January 2005), 21-42.

[5] Backus, David K., Allan W. Gregory and Stanley E. Zin, "Risk Premiums in the Term Structure: Evidence from Artificial Economies," Journal of Monetary Economics, 24, 3 (November 1989), 371-399.

[6] Bansal, Ravi and Amir Yaron, "Risks for the Long Run: A Potential Resolution of Asset Pricing Puzzles," Journal of Finance, 59, 4 (August 2004), 1481-1509.

[7] Campbell, John Y. and John H. Cochrane, "By Force of Habit: A Consumption-Based Explanation of Aggregate Stock Market Behavior," Journal of Political Economy, 107, 2 (April 1999), 205-251.

[8] Cecchetti, Stephen, Pok-sang Lam and Nelson Mark, "Mean Reversion in Equilibrium Asset Prices," American Economic Review, 80, 3 (June 1990), 398-418.

[9] Constantinides, George M., "Habit Formation: A Resolution of the Equity Premium Puzzle," Journal of Political Economy, 98, 3 (June 1990), 519-543.

[10] Fama, Eugene F. and Kenneth R. French, "Dividend Yields and Expected Stock Returns," Journal of Financial Economics 22, 1 (October 1988), 3-25. 
[11] Gali, Jordi, "Keeping up with the Joneses: Consumption Externalities, Portfolio Choice, and Asset Prices," Journal of Money, Credit and Banking, 26, 1 (February 1994), 18.

[12] Ibbotson Associates, Stocks, Bonds, Bills, Inflation, 1994 Yearbook, Ibbotson Associates, Chicago, 1994.

[13] Kandel, Shmuel and Robert F. Stambaugh, "Asset Returns and Intertemporal Preferences," Journal of Monetary Economics, 27,1 (February 1991) 39-71.

[14] Kocherlakota, Narayana R., "On the 'Discount' Factor in Growth Economies," Journal of Monetary Economics, 25, 1 (January 1990), 4347.

[15] Lettau, Martin, Sydney C. Ludvigson and Jessica A. Wachter, "The Declining Equity Premium: What Role Does Macroeconomic Risk Play?" April 2004.

[16] Lucas, Robert E., Jr., "Asset Prices in an Exchange Economy," Econometrica, 46, 6 (November 1978), 1429-1445.

[17] Mehra, Rajnish, "On the Existence and Representation of Equilibrium in an Economy with Growth and Nonstationary Consumption," International Economic Review, 29, 1 (February 1988), 131-135.

[18] Mehra, Rajnish and Edward C. Prescott, "The Equity Premium: A Puzzle," Journal of Monetary Economics, 15, 2 (March 1985), 145-161. 